\title{
AN ADAPTABLE DISCONTINUOUS GALERKIN SCHEME FOR THE WIGNER-FOKKER-PLANCK EQUATION*
}

\author{
IRENE M. GAMBA ${ }^{\dagger}$, MARIA PIA GUALDANI ${ }^{\ddagger}$, AND RICHARD W. SHARP§
}

\begin{abstract}
Recent analytic progress has increased demand for numerical approaches to the Wigner-Fokker-Planck (WFP) equation. We present a Discontinuous Galerkin scheme for the WFP equation with a general potential. Estimates showing convergence and stability of the scheme are provided. The scheme is adaptable, and may use both polynomial and non-polynomial basis functions.
\end{abstract}

Key words. Wigner-Fokker-Planck, discontinuous Galerkin

AMS subject classifications. $\quad 65 \mathrm{M} 12,35 \mathrm{Q} 40,65 \mathrm{M} 60,81 \mathrm{~V} 10,65 \mathrm{M} 15,65 \mathrm{Z} 05$.

\section{Introduction}

We propose a Discontinuous Galerkin (DG) method in order to numerically approximate the solution to the initial value problem for the time dependent WignerFokker-Planck (WFP) equation given a general smooth potential $V(x)$, posed for $(x, k) \in \mathbb{R}^{2 d}, t \in \mathbb{R}^{+}$

$$
w_{t}+k \cdot \nabla_{x} w+\Theta_{\hbar}[V](w)=Q_{\hbar, F P}(w) .
$$

The right hand side $Q_{\hbar, F P}(w)$ models the averaged environmental interactions with the system and is referred to as the Quantum Fokker-Planck operator. The operator $\Theta[V]$ is a pseudo-differential operator and takes into account the nonlocal action of the potential $V$.

In this paper we propose a Discontinuous Galerkin approximation for the above problem. The computation applies to a wide range of approximation spaces and does not rely on a basis of polynomials. We present also estimates showing convergence and stability of the scheme. The Discontinuous Galerkin (DG) approach proposed here provides several opportunities to optimize the approximation space. In particular, the use of non-polynomial basis functions, as proposed by Yuan and Shu in [28], allows for improvement over mesh refinement, increased polynomial order, and global or local basis set adjustments. The method is suitable to be adjusted to unstructured grids in space and time. The basis set may be a priori or adaptively optimized, depending on the specific circumstances of the calculation. Taken to the extreme, this allows the method to transition from a traditional DG solver to an essentially spectral solver. For example, to study perturbations of the harmonic potential one could use the known eigenfunctions of the harmonic case.

1.1. The model and related analytical results. Equation (1.1) is a kinetic quantum model for charge transport, used, for example, in the description of quantum Brownian motion, quantum optics, and plasma physics [12, 14, 13]. The function $w(x, k, t)$ is the Wigner transform of the density matrix $\rho(x, y, t)[27]$. It is Jin.

*Received: August 18, 2008; accepted (in revised version): May 20, 2009. Communicated by Shi

${ }^{\dagger}$ Department of Mathematics, The University of Texas at Austin (gamba@math.utexas.edu).

$\ddagger$ Department of Mathematics, The University of Texas at Austin (gualdani@math.utexas.edu).

$\S$ Department of Mathematical Sciences, Carnegie Mellon University, (rwsharp@cmu.edu). 
a quasi-probability function, which may take on negative values, and its zeroth and first moments with respect to $k$ produce, respectively, the nonnegative charge and flux densities associated with $\rho(x, t)$,

$$
\begin{aligned}
& \rho(x, t)=\int_{\mathbb{R}^{d}} w(x, k, t) d k, \\
& j(x, t)=\int_{\mathbb{R}^{d}} k w(x, k, t) d k .
\end{aligned}
$$

The Quantum Fokker-Planck term is a diffusion operator defined by

$$
Q_{\hbar, F P}(w)=D_{q q} \Delta_{x} w+2 \gamma \operatorname{div}_{k}(k w)+2 \frac{D_{p q}}{m} \operatorname{div}_{x}\left(\nabla_{k} w\right)+\frac{D_{p p}}{m^{2}} \Delta_{k} w
$$

The non-local pseudo-differential operator $\Theta[V]$ is defined as

$$
\Theta[V](w)=-\frac{i}{\hbar(2 \pi)^{d}} \int_{\mathbb{R}^{2 d}} \delta_{\hbar} V(x, \eta) w\left(x, k^{\prime}\right) e^{i \eta \cdot\left(k-k^{\prime}\right)} d k^{\prime} d \eta,
$$

with

$$
\delta_{\hbar} V(x, \eta)=V\left(x+\frac{\hbar}{m} \frac{\eta}{2}\right)-V\left(x-\frac{\hbar}{m} \frac{\eta}{2}\right)
$$

The coefficients $D_{q q}, \gamma, D_{p q}$, and $D_{p p}$ are constants that depend on several physical quantities. Specifically,

$$
D_{q q}=\frac{\lambda \hbar^{2}}{12 m^{2} k_{B} T} \quad \gamma=\frac{\lambda}{2 m} \quad D_{p q}=\frac{\lambda \Omega \hbar^{2}}{12 \pi m k_{B} T} \quad D_{p p}=\lambda k_{B} T,
$$

where $\hbar$ is Planck's constant, $m$ is particle mass, and $k_{B}$ is Boltzmann's constant. The operator is derived from a heat bath of harmonic oscillators, where $T$ is its temperature, $\lambda$ is the coupling constant, and $\Omega$ is the cut-off frequency. The constants satisfy the Lindblad condition: $D_{q q} D_{p p}-D_{p q}^{2} \geq \hbar^{2} \gamma^{2} / 4$, or equivalently $\Omega \leq k_{b} T / \hbar$. These conditions guarantee the quantum mechanically correct evolution of the system and convergence to the classical Fokker-Planck dynamics from stochastic calculus as $\hbar \rightarrow 0$. The reader is referred to $[6,12,21,25]$ for more details. In the following sections we actually work with the dimensionless version of the problem, although we use physical constants for the numerical simulations (specific values are noted in Section 4).

One may interpret the WFP equation as a quantum Liouville equation equated to an interaction operator $Q_{\hbar, F P}$ of Fokker-Planck type. When $Q_{\hbar, F P}:=0,(1.1)$ determines the time evolution of an isolated quantum system under the influence of a potential $V(x)$. This is equivalent to solving Schrödinger's equation, but the solution is a function of the $2 \mathrm{~d}$-dimensional phase space of the original problem. Despite increasing the dimensionality of the problem, the WFP equation offers the advantage of coupling the quantum system to its environment through $Q_{\hbar, F P}$. Specifically, (1.2) models the environmental interaction as a heat bath of harmonic oscillators [6].

It is known that in the semi-classical limit, $\hbar \rightarrow 0$, the interaction operator formally converges to $Q_{0, F P}=\Delta_{k} w+\operatorname{div}_{k}(k w)$, while the pseudo-differential operator simplifies to $\Theta_{\hbar}[V](w) \rightarrow-\nabla_{x} V \cdot \nabla_{k} w$. In particular, this limit yields the classical VlasovFokker-Planck equation with degenerate diffusion. Moreover, in the special case of the 
harmonic potential, $V(x)=\frac{1}{2}|x-a|^{2}$ with $a$ constant, the pseudo-differential operator of (1.3) has the same form as the classical acceleration term and can be rewritten,

$$
\Theta[V](w)=-(x-a) \cdot \nabla_{k} w \quad \text { for all } \hbar>0 .
$$

This provides a basis for comparison between the full WFP dynamics and known properties of the classical diffusion equation which was analyzed in [25]. This comparison results from balancing the classical transport operator for linear acceleration with the quantum corrected diffusion operator $Q_{\hbar, F P}$ defined in (1.2).

Numerous analytical results concerning the existence of local or global in time solutions to (1.1) are available, in the linear and nonlinear cases $[2,3,4,6,8,10]$. In the mean field approximation, when (1.1) is coupled to Poisson's equation for the electrostatic potential

$$
\Delta_{x} V(x, t)=-\int_{\mathbb{R}^{d}} w(x, k, t) d k,
$$

the nonlinear initial value problem has a classical solution for all time $t>0,[1,3,4]$.

Existence of global in time solutions associated to initial and boundary value problem in one space dimension for the Wigner-Poisson system $\left(Q_{\hbar, F P}=0\right)$ has been shown in [22], where the authors prove well-posedness in $C\left([0, \infty), L^{2}\left(\Omega_{x} \times \mathbb{R}_{k} ;(1+\right.\right.$ $\left.\left.k^{2}\right) d x d k\right)$ ), with inflow boundary conditions for the bounded spatial domain $\Omega_{x}$.

In addition to the local or global existence of solutions, there is the outstanding question of the existence of stationary states. For a harmonic potential $V(x)=\frac{1}{2} \mid x-$ $\left.a\right|^{2}$, existence of a smooth stationary solution to (1.1) in any space dimension is shown in [25]. The authors explicitly computed the unique stationary state $\mu(x, k)$ of (1.1) for $V(x)=|x|^{2} / 2$. Moreover it was shown in [25] that the corresponding time dependent solution, $w(x, k, t)$, to (1.1) with $V(x)=|x|^{2} / 2$ and general initial data $w_{I}$ exponentially decays in time toward the steady-state $\mu$.

Existence of stationary solutions to (1.1) for perturbations of harmonic potentials

$$
V(x)=\frac{|x|^{2}}{2}+V_{0}(x),
$$

is a subject of current investigation. A very recent result shows the existence, uniqueness, and convergence to a unique steady state, when the Lindblad condition holds, for sub-quadratic perturbations to the potential [5]. Current investigation also focuses on the case when $V_{0}(x)$ is a smooth regular function with control in its spectral norm. There it is supposed that there exists a unique steady-state, and the solution of the corresponding time-dependent problem converges exponentially to this steady-state, with the decay rate depending on the perturbation $V_{0}(x)$.

Although there has been recent theoretical progress on the WFP equation as contained in the previous references, few numerical simulations are available in cases where the interaction operator (1.2) is not included. Some of these approaches include splitting methods for the Wigner-Poisson problem $[7,26]$ and a finite difference approach to Wigner's equation [20]. Also, [19] developed convergence and spectral accuracy analysis of a semidiscrete version of the Wigner equation by means of a spectral method of a periodic approximation to the solution of the problem.

One recent technique for the complete Wigner-Fokker-Planck model (1.1)-(1.2) has been the use of continued-fraction methods by Garcia-Palacios and Zueco [17, 16]. The solution is approximated by expanding the Wigner function in a basis of Hermite polynomials of the momentum variable. Then a series of coupled equations for 
the evolution of the time and space dependent coefficients is derived. By design, the system of equations is such that individual equations are only coupled to their "neighbors," and the system may be solved by straightforward iteration. A difficulty arises in the treatment of arbitrary potentials which may complicate the structure of these equations and increase the number which need to be solved. Sharp potentials, such as a step potential, may be especially difficult to realize.

For comparison to the available analytical results, we work with the dimensionless version of the problem. To this end we set the terms $\gamma=\frac{1}{2}$ and $D_{p q}=0$ in (1.2), and the corresponding dimensionless initial value problem in $\mathbb{R}^{2 d} \times \mathbb{R}^{+}$becomes

$$
\begin{aligned}
& \left\{\begin{array}{c}
\partial_{t} w+k \cdot \nabla_{x} w+\Theta[V] w=\Delta_{k} w+\operatorname{div}_{k}(k w)+\Delta_{x} w \\
\left.w\right|_{t=0}=w_{I}(x, k),
\end{array}\right. \\
& \Theta[V](w)=-\frac{i}{(2 \pi)^{d}} \int_{\mathbb{R}^{2 d}} \delta V(x, \eta) w\left(x, k^{\prime}\right) e^{i \eta \cdot\left(k-k^{\prime}\right)} d k^{\prime} d \eta, \\
& \delta V(x, \eta)=V\left(x+\frac{\eta}{2}\right)-V\left(x-\frac{\eta}{2}\right) .
\end{aligned}
$$

These include the explicit formulas and associated decay rates analytically calculated in [25]. We briefly recall that the unique stationary state of (1.6) for $V(x)=|x|^{2} / 2$ reads as

$$
\mu(x, k)=\frac{1}{2 \sqrt{5} \pi} e^{-A(x, k)}, \quad A(x, k)=\left(\frac{1}{5}|x|^{2}+\frac{1}{5} x \cdot k+\frac{3}{10}|k|^{2}\right), \quad(x, k) \in \mathbb{R}^{2 d},
$$

and the exponential decay in time of the corresponding time dependent solution, $w(x, k, t)$, to (1.6) with $V(x)=|x|^{2} / 2$ and general initial data $w_{I}$ is given in the following norm,

$$
\left\|\frac{w-\mu}{\sqrt{\mu}}\right\|_{L^{2}\left(\mathbb{R}^{2 d}\right)} \leq e^{-\sigma t}\left\|\frac{w_{I}-\mu}{\sqrt{\mu}}\right\|_{L^{2}\left(\mathbb{R}^{2 d}\right)} .
$$

The value $\sigma$ is the largest positive constant such that $\operatorname{Hess}(A)-\sigma \mathbf{I} \geq 0$.

We have implemented the DG method using polynomial and non-polynomial approximation spaces in order to test its accuracy and efficiency. The method is based on a standard Non-symmetric Interior Penalty Galerkin (NIPG) treatment, but the pseudo-differential operator $\Theta[V](w)$ requires special attention. The form presented in (1.3) is ill-suited for numerical implementation. Practical representations of the operator are given, and the choice of which representation to use depends on the form of the potential. The various representations allow the method to accommodate a wide range of potential functions. Harmonic and sinusoidal potentials have compact exact representations which may be efficiently implemented. Also, methods for a few basic forms of the potential are quickly extended to linear combinations of the forms.

The pseudo-differential operator $\Theta[V]$ has been the main obstacle to both analytic and numerical studies of Wigner's equation. We implement three methods for evaluating $\Theta[V](w)$ in simulations. One is a general method, and the other two are efficient methods for specific forms of the potential $V$. It is difficult to work directly with the representation (1.3) in the DG framework because it doubles the degree of integration and although the overall integral is real valued, this is brought about by oscillatory cancellations (of course one need not explicitly evaluate the imaginary part, 
however, the real part is required and suffers the same oscillations). The efficiency and accuracy of the method can be greatly improved by finding alternate representations for $\Theta[V](w)$.

Of particular interest are perturbations of the harmonic potential,

$$
V(x)=\frac{|x-a|^{2}}{2}+V_{0}(x) .
$$

To treat non-harmonic potentials (i.e., the perturbation $V_{0}$ ), we use alternate representations of $\Theta[V]$. The basic approach is to take advantage of the well known properties of the Fourier transform to rewrite the pseudo-differential operator in convolution form,

$$
\begin{aligned}
\Theta[V](w) & =-\frac{i}{(2 \pi)^{d}} \int_{\mathbb{R}^{n}} \delta V(x, \eta) \mathcal{F}[w](x, \eta, t) \exp [i \eta \cdot k] d \eta \\
& =-\Im\left(\mathcal{F}^{-1}[\delta V](x, \cdot)\right) * w(x, \cdot, t),
\end{aligned}
$$

where we denote the Fourier transform in the variables $k^{\prime}$ and $\eta$ by

$$
\mathcal{F}[\varphi](\eta):=\int_{\mathbb{R}^{d}} \varphi\left(k^{\prime}\right) e^{-i k^{\prime} \cdot \eta} d k^{\prime}
$$

and the imaginary part of a value $z$ with $\Im(z)$. The function $\delta V$ is a real valued odd function in $\eta$, thus the real part of $\mathcal{F}^{-1}[\delta V]$ is zero. The expression is linear in $w$, real valued, and the degree of integration has been reduced. Given $\mathcal{F}^{-1}[\delta V]$, numerical evaluation of the convolution formula is practical. This is a method used for evaluating non-harmonic potentials. The final method is for the special case that the potential consists of a linear combination of sinusoidal functions. The inverse Fourier transform produces delta functions which simplify the convolution formula. This method is quite useful not just for potentials which are in Fourier series form, but also for potentials which can be locally approximated with sinusoidal functions (this includes polynomials). These methods allow us to treat many forms of potential functions, and several examples appear in Section 4.

The paper is organized as follows. Section 2 introduces the DG method for a general approximation space and our implementation for the WFP equation. Section 3 then presents estimates related to the convergence and stability of the method. These provide a basis to evaluate the resulting numerical method. Section 4 contains several numerical results which demonstrate agreement with known results from analysis, and additional results which go beyond the class of problems found in current analytic work. Section 5 contains concluding remarks, including an indication of how the availability of numerical simulations can and is being used to assist analytic progress.

\section{Implementation of the DG method}

The analytic results to which we would compare our numerical simulations are derived in all of $\mathbb{R}^{2 d}$, but we must compute on a finite domain. Numerically we will compute the solution of the WFP equation in a bounded domain with zero Dirichlet boundary conditions for both the $x$ and $k$ variables.

In this section we would like to justify the choice of the boundary conditions, showing that if the computational domain is large enough, the difference (in a certain norm specified later) between the solution of the boundary value problem and the one in the whole space does not increase with time, but stays small for all times. 
We proceed following the same approach presented in [15] for the analysis of the DG method for the linear Boltzmann equation.

First note that if the WFP in the whole space admits a stationary solution, $\mu(x, k)$ (e.g. (1.7) for the harmonic potential), which is integrable in $\mathbb{R}^{2 d}$ and normalized to unity, then

$$
\|\mu\|_{L_{\mu}^{2}(\Omega)}=\|\mu\|_{L^{1}(\Omega)}^{1 / 2}
$$

for any arbitrary set $\Omega$, where

$$
\|f\|_{L_{\mu}^{2}(\Omega)}^{2}:=\int_{\Omega} \frac{f^{2}}{\mu} d x .
$$

For arbitrary $\epsilon>0$, let $\Omega_{\epsilon} \subset \mathbb{R}^{2 d}$ be a set such that

$$
\int_{R^{2 d} \backslash \Omega_{\epsilon}}|\mu|<\epsilon
$$

Essentially, the set $\Omega_{\epsilon}$ is not "very big," because $w \in L_{\mu}^{2}$ means that $w^{2}$ decays quickly and is still integrable when multiplied by $\mu^{-1}(x, k)$.

We remark that, in addition to the harmonic case where (1.8) holds, the estimates in this section can be carried over for other potentials $V(x)$ under the assumption that there exists a unique stationary state $\mu \in L^{1}\left(\mathbb{R}^{2 d}\right)$ and the following inequality holds

$$
\|w(x, k, t)-\mu\|_{L_{\mu}^{2}\left(\mathbb{R}^{2 d}\right)} \leq g(t)\left\|w_{I}-\mu\right\|_{L_{\mu}^{2}\left(\mathbb{R}^{2 d}\right)},
$$

for any initial state $w_{I}$, where $g(t)$ is a positive and bounded function such that $\lim _{t \rightarrow \infty} g(t)=0$.

Now we estimate the solution $w$ in the cut-off domain $\Omega_{\epsilon}$. Since the solution $w(x, k, t)$ of $(1.2)$ is uniformly controlled in time and stable with respect to $w_{I}$ (see $(2.3))$, we can estimate the $L_{\mu}^{2}\left(\Omega_{\epsilon}\right)$-norm of the solution as follows:

$$
\|w\|_{L_{\mu}^{2}\left(\Omega_{\epsilon}\right)} \leq g(t)\left\|w_{I}-\mu\right\|_{L_{\mu}^{2}\left(\mathbb{R}^{2 d}\right)}+\|\mu\|_{L_{\mu}^{2}\left(\Omega_{\epsilon}\right)} \leq \mathbf{K}
$$

where the constant $\mathbf{K}$ is uniform in time. Similarly,

$$
\|w\|_{L_{\mu}^{2}\left(\mathbb{R}^{2 d} \backslash \Omega_{\epsilon}\right)} \leq g(t)\left\|w_{I}-\mu\right\|_{L_{\mu}^{2}\left(\mathbb{R}^{2 d}\right)}+\|\mu\|_{L_{\mu}^{2}\left(\mathbb{R}^{2 d} \backslash \Omega_{\epsilon}\right)}=C g(t)+\epsilon^{1 / 2},
$$

uniformly in time, where $C=\left\|w_{I}-\mu\right\|_{L_{\mu}^{2}\left(\mathbb{R}^{2 d}\right)}$, and $\|\mu\|_{L_{\mu}^{2}\left(\mathbb{R}^{2 d} \backslash \Omega_{\epsilon}\right)} \leq \epsilon^{1 / 2}$.

Since $\lim _{t \rightarrow \infty} g(t)=0$, there exists a time $T^{*}$ such that $C g\left(T^{*}\right)=\mathcal{O}\left(\epsilon^{1 / 2}\right)$. The value $T^{*}$ depends on the distance, in the $L_{\mu}^{2}$-norm, between the initial and stationary states, and on the decay rate $g(t)$. Consequently, for all $t>T^{*}$ it holds that

$$
\|w\|_{L_{\mu}^{2}\left(\mathbb{R}^{2 d} \backslash \Omega_{\epsilon}\right)} \leq \mathcal{O}\left(\epsilon^{1 / 2}\right) .
$$

Thus by choosing the domain $\Omega_{\epsilon}$ big enough, which means $\Omega_{\epsilon}$ contains almost all of the total mass of the initial datum $w_{I}$ and of the stationary states $\mu$, then (at least computationally, well beyond machine accuracy) the solution of the Cauchy problem $w$ at the boundary of $\Omega_{\epsilon}$ will be zero, with zero derivatives, and the associated evolution problem will essentially be confined to the domain $\Omega_{\epsilon}$. 
To the best of our knowledge, there is no available analytical result at the present time to rigorously justify this last statement, which is an assumption for the initial boundary value problem under consideration and the corresponding one in all space.

We remind the reader that the above estimates do not provide a pointwise control of the solution to (1.2) outside the domain $\Omega_{\epsilon}$, but give a nice estimate in the $L^{2}$ norm.

It is important to note that this approach is intended to heuristically justify the selection of the computational domain. However, the calculation of error estimates in the following sections are with respect to the solution of the initial value problem in the bounded domain.

Our aim is to produce a discrete approximation to the solution of the initial value problem (1.6) in $\Omega \times \mathbb{R}^{+}$, for a bounded domain $\Omega$ with homogeneous Dirichlet boundary conditions. The numerical simulations presented in section 4 use $d=1$, but neither the construction of the method nor its analysis depend on this particular choice. The computational domain $\Omega \subset R^{2}$ is the following: consider $\Omega=\Omega^{x} \times \Omega^{k} \subset \mathbb{R}^{2}$, where $\Omega^{x}:=[0, L] \subset \mathbb{R}$ and $\Omega^{k}:=[-K, K] \subset \mathbb{R}$. The boundaries of $\Omega$ are defined by

$$
\begin{aligned}
\partial \Omega^{x} & :=\left\{(x,-K) \mid \forall x \in \Omega^{x}\right\} \cup\left\{(x, K) \mid \forall x \in \Omega^{x}\right\}, \\
\partial \Omega^{k}:=\partial \Omega^{0} \cup \partial \Omega^{L} & :=\left\{(0, k) \mid \forall k \in \Omega^{k}\right\} \cup\left\{(L, k) \mid \forall k \in \Omega^{k}\right\},
\end{aligned}
$$

and are depicted in figure 2.1. The homogeneous Dirichlet boundary conditions on $\partial \Omega^{x}$ and $\partial \Omega^{k}$ for the associated boundary value problem to (1.6) are defined by

$$
\begin{array}{r}
w(x, K, t)=w(x,-K, t)=0 \text { on } \partial \Omega^{x} \\
w(0, k, t)=w(L, k, t)=0 \text { on } \partial \Omega^{k} .
\end{array}
$$

2.1. Notation. The domain $\Omega$ is partitioned into mutually disjoint open subsets (or cells) $\Omega_{j}=\Omega_{j}^{x} \times \Omega_{j}^{k}$, where the point $(x, k) \in \Omega, x \in \Omega_{j}^{x}$ and $k \in \Omega_{j}^{k}$. Let $h_{j}$ be the diameter of $\Omega_{j}$, and let $h$ be the maximum diameter over the cells in this nondegenerate subdivision of the domain $\Omega_{h}^{N}=\left\{\Omega_{j}\right\}_{j=1}^{N}$. Moreover, let $E_{j}=$ $\left\{e_{(j, \zeta)}\right\}_{\zeta=1}^{F_{j}}$ be the set of faces belonging to $\partial \Omega_{j}$, the boundary of $\Omega_{j}$. If $E_{j} \cap \partial \Omega \neq \emptyset$, then any face $e_{(j, \zeta)} \in E_{j} \cap \partial \Omega$ is an exterior face, which will be indicated by writing $\partial e_{(j, \zeta)}$. Moreover, let $E^{e}=\cup_{j} E_{j} \backslash \partial \Omega$ be the set of all internal faces, thus partitioning the set of all faces, $E^{h}=E^{e} \cup \partial \Omega, E^{e} \cap \partial \Omega=\emptyset$. To each face $e_{(j, \zeta)}$ we associate an outward facing unit normal vector $\nu_{(j, \zeta)}$ such that $\nu_{(j, \zeta)}$ coincides with $\nu$ on $\partial \Omega$. Note that any internal face corresponds to two cells $\Omega_{j}$ and $\Omega_{j^{\prime}}$, so for some pairs $\left(j^{\prime}, \zeta^{\prime}\right), e_{(j, \zeta)}=e_{\left(j^{\prime}, \zeta^{\prime}\right)}$. Summation of faces over the double index would count each face twice, so sometimes it is more convenient to use a single index to identify a particular face, $e_{i} \in E$, without reference to a specific cell. The normal vectors are indexed similarly, however, face $\zeta$ of cell $j$ has a unique outward pointing normal, $\nu_{(j, \zeta)}$, which is antiparallel to the corresponding $\nu_{\left(j^{\prime}, \zeta^{\prime}\right)}$. Thus, $e_{(j, \zeta)}$ and $e_{\left(j^{\prime}, \zeta^{\prime}\right)}$ may not be simply interchangeable in expressions which depend on the normal vectors. This is the case (implicitly) in the following definitions for jump and average operators: for any interior face $e_{(1, \zeta)} \in \partial \Omega_{1} \cap \partial \Omega_{2}$ we define the jump, [.], and average, $\{\cdot\}$, of a function $f$ across the edge,

$$
\begin{aligned}
{[f]: } & =\left.f\right|_{\Omega_{1}}-\left.f\right|_{\Omega_{2}}, \\
\{f\} & :=\frac{\left.f\right|_{\Omega_{1}}+\left.f\right|_{\Omega_{2}}}{2} .
\end{aligned}
$$




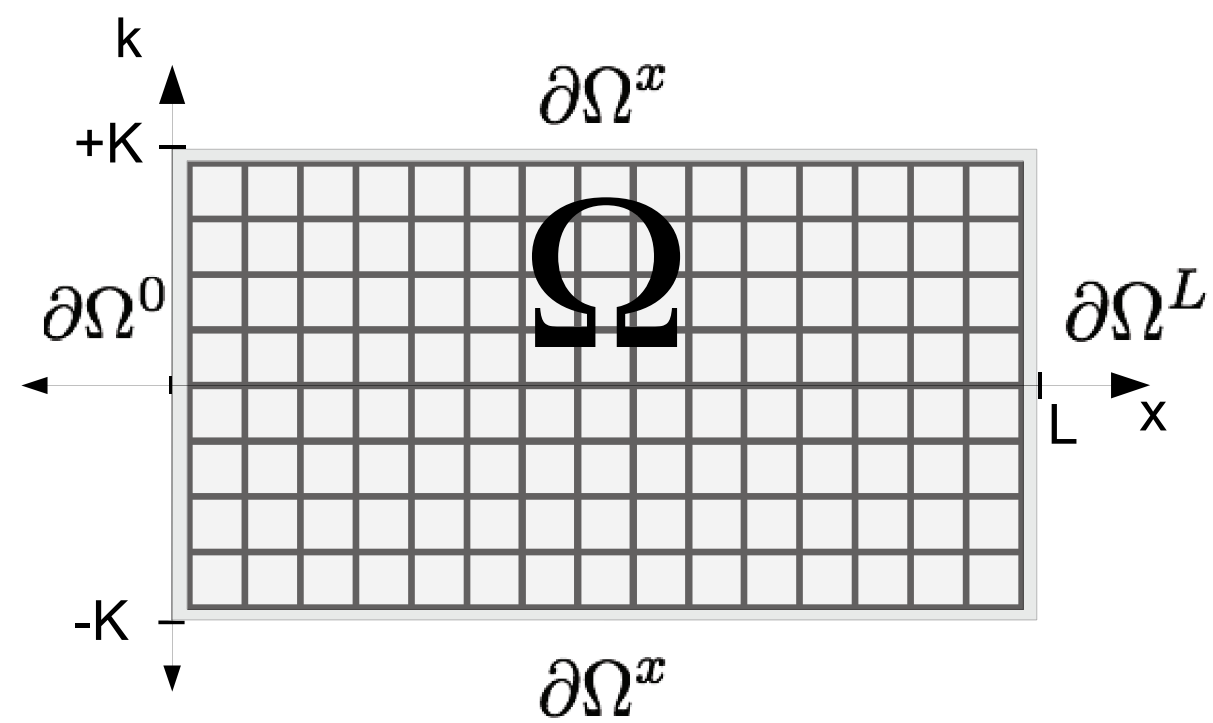

Figure 2.1. A schematic of the domain for $d=1$.

The following identity is used frequently,

$$
[f g]=[f]\{g\}+\{f\}[g] .
$$

Another notation used at cell boundaries denotes the upwind value of a function $f$,

$$
f^{\uparrow}=\left.f\right|_{\Omega_{1}} \chi_{\left[\alpha \cdot \nu_{k}>0\right]}+\left.f\right|_{\Omega_{2}} \chi_{\left[\alpha \cdot \nu_{k}<0\right]},
$$

where $\chi_{[\cdot]}$ is the characteristic function and $\operatorname{sign}(\alpha \cdot \nu)$ determines the upwind direction.

The discrete approximation $w_{h}$ is an element of some approximation space $\mathcal{V}$. The approximation spaces used in this work are produced by basis functions $\phi(x, k)$, which are themselves products of primitive basis functions $\varphi(x)$ and $\psi(k)$. Each basis function is compactly supported on a single cell, and all primitive basis functions (and therefore their products) are mutually orthogonal. The orthogonality of the basis functions is a purely practical consideration, and not a requirement of the DG approach. The approximation spaces used in the simulations presented in Section 4 (where $d=1$ ) are,

$$
\begin{aligned}
& \mathcal{V}_{\mathcal{P}}^{p}:=\operatorname{span}\left\{\phi_{(j, m)}(x, k)=\varphi(x) \psi(k) \mid \varphi, \psi \in \mathcal{P}_{\Omega_{j}}^{p}\right\}, \\
& \mathcal{V}_{T}:=\operatorname{span}\left\{\phi_{(j, m)}(x, k)=\varphi(x) \psi(k) \mid \varphi, \psi \in\{1, \sin (\omega(s-\delta)), \cos (\sigma(s-\delta))\}\right\}, \\
& \mathcal{V}_{H}^{p}:=\operatorname{span}\left\{\phi_{(j, m)}(x, k)=\varphi(x) \psi(k) \mid \varphi, \psi \in H_{\Omega_{j}}^{p}\right\} .
\end{aligned}
$$

The double index $(j, m)$ identifies the cell which supports the basis function, $\Omega_{j}$, and the $m^{\text {th }}$ basis function on that cell. As with cell faces, sometimes it will be 
more appropriate to identify basis functions with a single index, $\phi_{n}$, that does not specifically identify a particular cell. The functions $\mathcal{P}_{\Omega_{j}}^{p}$ are Legendre polynomials, up to degree $p$, which have been shifted and scaled so that their typical orthogonality relation holds on each cell, i.e., $\Omega_{j}^{x} \varphi_{(j, m)}(x) \varphi_{\left(j, m^{\prime}\right)}(x) d x=\delta_{m, m^{\prime}}$, and similarly for $\psi(k)$ over $\Omega_{j}^{k}$. In $\mathcal{V}_{T}$ the frequencies $\omega$ and $\sigma$ are chosen so that the width of the cell is an integer multiple of the wavelength of the function. Finally, the functions in $H_{\Omega_{j}}^{p}$ are Hermite functions, up to degree $p$, which have been restricted (not rescaled) to cell $\Omega_{j}$ and then locally orthogonalized using the Gram-Schmidt procedure. With respect to any approximation space, we write $w_{A}^{*}$ to denote the continuous interpolant of $w$ in $\mathcal{V}_{A} \cap C^{0}(\Omega)$.

2.2. Implementation. The function $w_{h}$ approximates $w$ and is a linear combination of the elements of $\mathcal{V}_{A}$. The computational task is to calculate the time evolution of the expansion coefficients, $c_{n}(t)$,

$$
w(x, k, t) \approx w_{h}(x, k, t)=\sum_{n} c_{n}(t) \phi_{n}(x, k) .
$$

We rewrite (1.1) as

$$
w_{t}=F(w) .
$$

The problem of finding the semi-discrete discontinuous Galerkin approximation to (2.6) in a bounded domain $\Omega$ is: seek $w_{h}(x, k, t) \in \mathbb{R}_{t}^{+} \times \mathcal{V}_{A}$ such that,

$$
w_{h}(\cdot, \cdot, t)=0, \quad \text { on } \partial \Omega, \quad w_{h}(x, k, 0)=\mathbb{P}_{\mathcal{V}_{A}} w_{I}(x, k),
$$

and, for all $t>0$, it holds that

$$
\left(w_{h t}, \psi_{h}\right)_{\Omega}=\left(F\left(w_{h}\right), \psi_{h}\right)_{\Omega}, \quad \forall \psi_{h} \in \mathcal{V}_{A},
$$

where $(\cdot, \cdot)_{\Omega}$ denotes the standard scalar product in $L^{2}(\Omega)$ and $\mathbb{P}_{\mathcal{V}_{A}}$ the projection on the approximated space $\mathcal{V}_{A}$.

The weak formulation becomes,

$$
\sum_{n} \frac{d}{d t} c_{n}(t)\left(\phi_{n}, \psi_{h}\right)_{\Omega}=\left(F\left(\sum_{n} c_{n}(t) \phi_{n}\right), \psi_{h}\right)_{\Omega} .
$$

Choosing $\psi_{h}=\phi_{p}$, a decoupled system of ODEs for the time dependent coefficients $c_{p}(t)$ is produced (revealing the practical reason for using mutually orthogonal basis functions),

$$
\frac{d}{d t} c_{p}(t)=\frac{\left(F\left(\sum_{n} c_{n}(t) \phi_{n}\right), \phi_{p}\right)_{\Omega}}{\left(\phi_{p}, \phi_{p}\right)_{\Omega}}=\tilde{F}_{p}(c(t)), \quad \forall k .
$$

A standard third-order total variation diminishing Runge-Kutta method is used to solve this system [11, 18, 24],

$$
\begin{aligned}
c^{(1)} & =c^{t}+\Delta t \tilde{F}\left(c^{t}\right), \\
c^{(2)} & =\frac{3}{4} c^{t}+\frac{1}{4} c^{(1)}+\frac{1}{4} \Delta t \tilde{F}\left(c^{(1)}\right), \\
c^{t+\Delta t} & =\frac{1}{3} c^{t}+\frac{2}{3} c^{(2)}+\frac{2}{3} \Delta t \tilde{F}\left(c^{(2)}\right) .
\end{aligned}
$$


The right hand side, $\tilde{F}(c(t))$, is discretized as follows: let $V(x)=\frac{1}{2}|x|^{2}+V_{0}(x)$, where $V_{0}(x) \in W_{\infty}^{1}\left(\mathbb{R}^{d}\right)$. Equation (2.6) can be rewritten as

$$
\int_{\Omega} \partial_{t} w_{h} \psi_{h} d x+\left(\Theta\left[V_{0}\right]\left(w_{h}\right), \psi_{h}\right)_{L^{2}(\Omega)}=\left(L w_{h}, \psi_{h}\right)_{L^{2}(\Omega)}
$$

where $L$ is the linear operator

$$
L w:=-k \cdot \nabla_{x} w+x \cdot \nabla_{k} w+2 \operatorname{div}_{k}(k w)+\Delta_{x} w+\Delta_{k} w .
$$

The alert reader will recognize (1.6), but with a factor of two in front of the divergence term. In the analysis we use the factor of 2 to be consistent with previous works, but in the numerical simulations we change this value to 1 . The analytic stationary state reported in section 1 coincides with the value 1 , and is the stationary state which will be used for comparison in the numerical section. In this and the next section we will use the value 2 . The bilinear expression for $L w$ and the test function is

$$
\begin{aligned}
(L w, \psi)_{L^{2}(\Omega)}= & \sum_{\Omega_{j} \in \Omega_{h}^{N}}(w, \alpha \cdot \nabla \psi)_{\Omega_{j}}-\sum_{e_{i} \in E^{e}}\left\langle w^{\uparrow}[\psi], \alpha \cdot \nu_{i}\right\rangle_{e_{i}} \\
& -\langle w \psi, \alpha \cdot \nu\rangle_{\partial \Omega}-\sum_{\Omega_{j} \in \Omega_{h}^{N}}(\nabla \psi, \nabla w)_{\Omega_{j}} \\
& +\sum_{e_{i} \in E^{e}}\left\langle[\psi],\left\{\nabla w \cdot \nu_{i}\right\}\right\rangle_{e_{i}}-\sum_{e_{i} \in E^{e}}\left\langle[w],\left\{\nabla \psi \cdot \nu_{i}\right\}\right\rangle_{e_{i}} \\
& +\langle\psi, \nabla w \cdot \nu\rangle_{\partial \Omega}-\sum_{e_{i} \in E^{e}} \frac{1}{\left|e_{i}\right|}\langle[w],[\psi]\rangle_{e_{i}},
\end{aligned}
$$

with $\alpha:=(k,-x-2 k), \nabla:=\left(\begin{array}{c}\nabla_{x} \\ \nabla_{k}\end{array}\right)$, and $\left|e_{i}\right|$ denotes the length of the face $e_{i}$. The pseudo-differential term is

$$
\begin{aligned}
& \left(\Theta\left[V_{0}\right](w), \psi\right)_{L^{2}\left(\Omega_{h}\right)} \\
= & -\frac{i}{(2 \pi)^{d}} \int_{\Omega_{h}} \psi(x, k, t)\left(\int_{\mathbb{R}^{2 d}} \delta V_{0}(x, \eta) w\left(x, k^{\prime}, t\right) e^{i \eta \cdot\left(k-k^{\prime}\right)} d k^{\prime} d \eta\right) d x d k
\end{aligned}
$$

In order for the nonlocal operator $\Theta\left[V_{0}\right]$ to be well defined, the function $w_{h}$ is meant to be extended to the whole space in the variable $k$ by the value zero.

The notation $\langle\cdot, \cdot\rangle_{e_{i}}$ represents the integration of boundary terms over a cell face following integration by parts in $x$ or $k$. The Dirichlet boundary conditions (2.5) of the problem are enforced for the boundary terms $\langle w \psi, \alpha \cdot \nu\rangle_{\partial \Omega}$ and $\langle\psi, \nabla w \cdot \nu\rangle_{\partial \Omega}$ through the identities

$$
\langle w \psi, \alpha \cdot \nu\rangle_{\partial \Omega}=\frac{1}{2}\langle w \psi, \alpha \cdot \nu\rangle_{\partial \Omega}
$$

and

$$
\langle\psi, \nabla w \cdot \nu\rangle_{\partial \Omega}=\langle\psi, \nabla w \cdot \nu\rangle_{\partial \Omega}-\langle w, \nabla \psi \cdot \nu\rangle_{\partial \Omega}
$$


The weak formulation of the problem is:

$$
\begin{gathered}
\left(\partial_{t} w_{h}, \psi_{h}\right)_{\Omega}-\sum_{\Omega_{j} \in \Omega_{h}^{N}}\left(w_{h}, \alpha \cdot \nabla \psi_{h}\right)_{\Omega_{j}}+\sum_{e_{i} \in E^{e}}\left\langle w_{h}^{\uparrow}\left[\psi_{h}\right], \alpha \cdot \nu_{i}\right\rangle_{e_{i}}+\frac{1}{2}\left\langle w_{h} \psi_{h}, \alpha \cdot \nu\right\rangle_{\partial \Omega} \\
+\left(\Theta\left[V_{0}\right]\left(w_{h}\right), \psi_{h}\right)_{\Omega} \\
=-\sum_{\Omega_{j} \in \Omega_{h}^{N}}\left(\nabla \psi_{h}, \nabla w_{h}\right)_{\Omega_{j}}+\sum_{e_{i} \in E^{e}}\left\langle\left[\psi_{h}\right],\left\{\nabla w_{h} \cdot \nu_{i}\right\}\right\rangle_{e_{i}}-\sum_{e_{i} \in E^{e}}\left\langle\left[w_{h}\right],\left\{\nabla \psi_{h} \cdot \nu_{i}\right\}\right\rangle_{e_{i}} \\
+\left\langle\psi_{h}, \nabla w_{h} \cdot \nu\right\rangle_{\partial \Omega}-\left\langle w_{h}, \nabla \psi_{h} \cdot \nu\right\rangle_{\partial \Omega}-\sum_{e_{i} \in E^{e}} \frac{1}{\left|e_{i}\right|}\left\langle\left[w_{h}\right],\left[\psi_{h}\right]\right\rangle_{e_{i}} .
\end{gathered}
$$

The basic integrals, those actually computed numerically, are determined by selecting the elements of $\left\{\phi_{p}\right\}$ as test functions. Let $\left\{\phi_{p}\right\}$ be a test function with support in the cell $\Omega_{j}$, then

$$
\begin{aligned}
\left(w_{h}, \alpha \cdot \nabla \phi_{p}\right)_{\Omega_{j}} & =\sum_{n \in \Omega_{j}} c_{n}\left(\phi_{n}, \alpha \cdot \nabla \phi_{p}\right)_{\Omega_{j}}, \\
\left\langle w_{h}^{\uparrow}\left[\phi_{p}\right], \alpha \cdot \nu_{i}\right\rangle_{e_{i}} & =\delta_{e_{i} \in \partial \Omega_{j}} \sum_{n \in \partial \Omega_{j}} c_{n}\left\langle(\alpha \cdot \nu) \phi_{n}^{\uparrow}, \phi_{p}\right\rangle_{e_{i}}, \\
\left\langle\left[w_{h}\right],\left\{\nabla \phi_{p} \cdot \nu_{i}\right\}\right\rangle_{e_{i}} & =\frac{\delta_{e_{i} \in \partial \Omega_{j}}}{2} \sum_{n \in \partial \Omega_{j}} c_{n}\left\langle\phi_{n}, \nabla \phi_{p} \cdot \nu\right\rangle_{e_{i}}, \\
\left\langle\left[\phi_{p}\right],\left\{\nabla w_{h} \cdot \nu_{i}\right\}\right\rangle_{e_{i}} & =\frac{\delta_{e_{i} \in \partial \Omega_{j}}}{2} \sum_{n \in \partial \Omega_{j}} c_{n}\left\langle\nabla \phi_{n} \cdot \nu, \phi_{p}\right\rangle_{e_{i}}, \\
\left(\nabla \phi_{p}, \nabla w_{h}\right)_{\Omega_{j}} & =\sum_{n \in \Omega_{j}} c_{n}\left(\nabla \phi_{n}, \nabla \phi_{p}\right)_{\Omega_{j}} .
\end{aligned}
$$

The notation $n \in \Omega_{j}$ indicates that the sum is only over the basis functions $\phi_{n}$ which have support on cell $\Omega_{j}$. While mutual orthogonality is no longer expected ( $\alpha$ depends on $k$ and we have differentiated), a pair of basis functions still must share the same supporting cell to produce a nonzero integral. Similarly, in the second expression, the functions $\phi_{n}$ and $\phi_{p}$ must be supported on cells which share a face, $n \in \partial \Omega_{j}$. The face in question, $e_{i}$ must also be one of the faces of $\partial \Omega_{j}$, indicated by the term $\delta_{e_{i} \in \partial \Omega_{j}}$, which is 1 if this is true and 0 otherwise. In the next section, there is also reference to $n \in \Omega_{j(p)}$, meaning the set of all $n$ such that $\operatorname{supp} \phi_{n} \cap \operatorname{supp} \phi_{p} \neq \emptyset$ given $\phi_{p}$.

The WFP equation (1.1) is mass-conserving, in the sense that

$$
\int_{\mathbb{R}^{2 d}} w(x, k, t) d x d k=\text { const. } \quad \forall t \geq 0 .
$$

This conservation property does not hold if the problem is considered in a bounded domain with zero-Dirichlet boundary conditions. The scheme presented here, for (1.1) with homogeneous Dirichlet boundary condition, is not mass conserving. However if the basis functions are piecewise constants, one may easily check that the above scheme is mass conserving.

This property will be shown in the numerical simulations in Section 4 . 
2.3. The pseudo-differential operator. The pseudo-differential operator is evaluated in one of three ways depending on the specific form of the potential. The simplest is the approach used for the harmonic potential. As noted in the introduction, the form of the pseudo-differential operator in this case is $-(x-a) \cdot \nabla_{k} w$. In the DG scheme this term is treated analogously to the transport term, $k \cdot \nabla_{x} w$.

The second approach is to work directly with the convolution form of the operator,

$$
\Theta[V](w)=-\Im\left(\mathcal{F}^{-1}[\delta V](x, \cdot)\right) * w(x, \cdot, t) .
$$

In bilinear form, using $\phi_{p}$ as the test function, all of the required integrals are restricted to finite intervals

$$
\begin{aligned}
& \left(\Theta[V]\left(w_{h}\right), \phi_{p}(x, k)\right)_{\Omega} \\
= & -\sum_{n \in \Omega_{j(p)}^{x}} c_{n} \int_{\Omega_{j(p)}^{k}} \int_{\Omega_{j(p)}^{x}} \int_{\Omega_{j(n)}^{k}} \Im\left(\mathcal{F}^{-1}[\delta V](x, k-s)\right) \phi_{n}(x, s) \phi_{p}(x, k) d s d x d k .
\end{aligned}
$$

The sum is now carried out over all $n \in \Omega_{j(p)}^{x}$, which indicates all functions $\phi_{n}$ with support in $\Omega_{j(p)}^{x} \times\left(\cup_{z} \Omega_{z}^{k}\right)$, that is, over all $n$ such that the spatial component of the support of $\phi_{n}$ overlaps the spatial component of the support of $\phi_{p}$. Thus, the integral in $s$ is restricted by the support of $\phi_{n}$ and the integrals in $x$ and $k$ are restricted by the support of $\phi_{p}$. Although the integrals may be oscillatory due to the inverse transform of the potential, these oscillations are independent of the mesh and so could be eliminated by mesh refinement.

This approach is practical when the inverse Fourier transform of the potential is available and easily integrated by numerical methods. Gaussian potentials provide one useful and obvious example. Step potentials (Heaviside functions) are also treated in this manner. For the potential,

$$
V_{a}(x)=\left\{\begin{array}{cc}
0 & x<a \\
\frac{1}{2} & x=a \\
1 & x>a
\end{array}\right\},
$$

the inverse Fourier transform of $\delta V$ is,

$$
\mathcal{F}^{-1}[\delta V](x, k)=i \frac{\cos (2(x-a) k)}{\pi k} .
$$

Linear combinations of step potentials are used in section 4 to produce numerical examples in which $w$ is confined to a bin constructed of square walls with finite height and width.

Finally, the convolution approach can be further streamlined if there is a closed form for the convolution. This is possible for the important case of sinusoidal potentials. The inverse Fourier transform then produces delta functions, and the convolution may be evaluated immediately. Suppose $V(x)=\sum_{a} v_{a} \exp [i x a 2 \pi / P]$; then the function $\delta V(x, \eta)$ is

$$
\begin{aligned}
\delta V(x, \eta) & =\sum_{a} v_{a} \exp [i a x 2 \pi / P](\exp [i \eta a \pi / P]-\exp [-i \eta a \pi / P]) \\
& =\sum_{a} 2 v_{a} \exp \left[i\left(\frac{2 \pi a x}{P}+\frac{\pi}{2}\right)\right] \sin (\pi a \eta / P) .
\end{aligned}
$$


Applying the inverse Fourier transform, one arrives at the expression

$$
-\Im\left(\mathcal{F}^{-1}[\delta V](x, k)\right)=\sum_{a} \tilde{v}_{a}(x)(\delta(k-a \pi / P)-\delta(k+a \pi / P)),
$$

where the real valued coefficients $\tilde{v}_{a}(x)$ are

$$
\tilde{v}_{a}(x)=2 \pi\left(\Im\left(v_{a}\right) \cos (2 \pi a x / P)+\Re\left(v_{a}\right) \sin (2 \pi a x / P)\right) .
$$

Applying convolution,

$$
\begin{aligned}
\Theta[V](w(x, k, t)) & =-\Im\left(\mathcal{F}^{-1}[\delta V](x, \cdot)\right) * w(x, \cdot, t) \\
& =\sum_{a} \tilde{v}_{a}(x)((\delta(k-a \pi / P)-\delta(k+a \pi / P)) * w(x, \cdot, t)) \\
& =\sum_{a} \tilde{v}_{a}(x)(w(x, k-a \pi / P, t)-w(x, k+a \pi / P, t)) .
\end{aligned}
$$

Replacing $w$ with $w_{h}$ and selecting basis function $\phi_{p}(x, k)$ as the test function, the basic integrals to be computed are

$$
\left(\Theta[V]\left(w_{h}\right), \phi_{p}\right)=\sum_{n \in \Omega_{j(p)}} c_{n}\left(\sum_{a} \tilde{v}_{a}(x) \phi_{n}(x, k \pm a \pi / P), \phi_{p}(x, k)\right)_{\Omega_{j}} .
$$

Note that the nonlocal nature of the operator means that the support of $\phi_{n}$ does not need to be the same as the support of $\phi_{p}$ to produce a nonzero result. No boundary terms arise, but integration is nonlocal, and one must calculate the coefficients $\tilde{v}_{a}(x)$. The accuracy of the representation depends on the finite number of terms that one is able to afford to compute. Fortunately, many useful potentials may be accurately, even exactly represented with just a few terms. Primus inter pares:

$$
V(x)=\alpha \sin (x+\theta)+\gamma,
$$

where $\alpha, \theta$, and $\gamma$, are constants. For this family of potentials, $\Theta[V](w)$ has exact, two term Fourier representations. However, even for functions which require more Fourier terms, there is only a linearly growing computational cost. No additional theoretical development is needed (as would have been the case for the differential representation).

A wide range of potentials in the form of (1.5) may be treated by combining the two representations of $\Theta[V]$. Even polynomials such as $x^{2}$ may be approximated locally using a combination of sinusoidal terms. This example provides a useful test for verifying the numerical implementation, and is included in the results in section 4 . Between the special method for harmonic potentials, the convolution method, and the sinusoidal method, a wide range of potential functions, and any linear combination thereof, is available.

\section{Analysis of the numerical scheme}

3.1. Interpolation and approximation results. We briefly recall some approximation results that will be useful: 
LEMMA 3.1. Let $\Omega_{h}$ be a simply connected subset of $\Omega \subset \mathbb{R}^{d}$ with $h=\operatorname{diam}\left(\Omega_{h}\right)$ and $e$ be a face of $\Omega_{h}$ with normal vector $\nu$. If $p_{h}$ is a finite polynomial on $\Omega_{h}$ there exists a constant $c$, not depending on $\Omega_{h}$, such that

$$
\begin{aligned}
\left\|p_{h}\right\|_{2, e} & \leq \frac{c}{\sqrt{h}}\left\|p_{h}\right\|_{2, \Omega_{h}}, \\
\left\|\nabla p_{h} \cdot \nu\right\|_{2, e} & \leq \frac{c}{\sqrt{h}}\left\|\nabla p_{h}\right\|_{2, \Omega_{h}}, \\
\left\|p_{h}\right\|_{H^{1}\left(\Omega_{h}\right)} & \leq \frac{c}{h}\left\|p_{h}\right\|_{2, \Omega_{h}} .
\end{aligned}
$$

Proof. See [23].

Furthermore, for each $f(x, t) \in H^{2}(\Omega)$ and its continuous interpolant $f_{P}^{*}(x, t) \in$ $\mathcal{V}_{P} \cap C^{0}(\Omega)$, the following approximation properties hold for all $t>0$ for $q=0,1,2$ :

$$
\begin{aligned}
\left\|f(\cdot, t)-f_{P}^{*}(\cdot, t)\right\|_{H^{q}\left(\Omega_{h}\right)} & \leq c h^{p+1-q}\|f(\cdot, t)\|_{H^{p+1}\left(\Omega_{h}\right)}, \\
\left\|\partial_{t} f(\cdot, t)-\partial_{t} f_{P}^{*}(\cdot, t)\right\|_{2, \Omega_{h}} & \leq c h^{p}\left\|\partial_{t} f(\cdot, t)\right\|_{H^{p}\left(\Omega_{h}\right)} .
\end{aligned}
$$

We define the element space

$$
\mathcal{V}_{P}:=\left\{\varphi \mid \varphi_{\left.\right|_{\Omega_{j}}} \text { polynomial of total degree less than or equal to } \mathrm{p}\right\}
$$

and denote with $w_{P}^{*}$ the continuous interpolant of $w$ in $\mathcal{V}_{P} \cap C^{0}(\Omega)$ (note that $\mathcal{V}_{P}$ is distinct from the orthogonalized version $\mathcal{V}_{\mathcal{P}}^{p}$ mentioned in Section 2).

3.2. Stability, consistency and $L^{2}$-error estimates. Let $V(x)=\frac{1}{2}|x|^{2}+$ $V_{0}(x)$, where $V_{0}(x) \in W_{\infty}^{1}\left(\mathbb{R}^{d}\right)$. Again, the weak formulation of the problem is

$$
\begin{aligned}
& \left(\partial_{t} w_{h}, \psi_{h}\right)_{\Omega}-\sum_{\Omega_{h}}\left(w_{h}, \alpha \cdot \nabla \psi_{h}\right)_{\Omega_{h}}+\sum_{e_{i} \in E^{e}}\left\langle w_{h}^{\uparrow}\left[\psi_{h}\right], \alpha \cdot \nu_{i}\right\rangle_{e_{i}}+\frac{1}{2}\left\langle w_{h} \psi_{h}, \alpha \cdot \nu\right\rangle_{\partial \Omega} \\
& +\left(\Theta\left[V_{0}\right]\left(w_{h}\right), \psi_{h}\right)_{\Omega} \\
= & -\sum_{\Omega_{h}}\left(\nabla \psi_{h}, \nabla w_{h}\right)_{\Omega_{h}}+\sum_{e_{i} \in E^{e}}\left\langle\left[\psi_{h}\right],\left\{\nabla w_{h} \cdot \nu_{i}\right\}\right\rangle_{e_{i}}-\sum_{e_{i} \in E^{e}}\left\langle\left[w_{h}\right],\left\{\nabla \psi_{h} \cdot \nu_{i}\right\}\right\rangle_{e_{i}} \\
& +\left\langle\psi_{h}, \nabla w_{h} \cdot \nu\right\rangle_{\partial \Omega}-\left\langle w_{h}, \nabla \psi_{h} \cdot \nu\right\rangle_{\partial \Omega}-\sum_{e_{i} \in E^{e}} \frac{1}{\left|e_{i}\right|}\left\langle\left[w_{h}\right],\left[\psi_{h}\right]\right\rangle_{e_{i}},
\end{aligned}
$$

with $\Omega \subset \mathbb{R}^{2 d}$ and where summation over $\Omega_{j} \in \Omega_{h}^{N}$ is now summation over $\Omega_{h}$ for consistency with Lemma 3.1, and reflecting the significance of $h$. In this section we will make use of the following identities: for all functions $f \in \mathcal{V}_{P}$, we have

$$
\frac{1}{2}\left\langle\left[f^{2}\right], \alpha \cdot \nu\right\rangle_{e}-\left\langle f^{\uparrow}[f], \alpha \cdot \nu_{i}\right\rangle_{e}=-\frac{1}{2}\left\langle[f]^{2},|\alpha \cdot \nu|\right\rangle_{e}
$$

where $e$ denotes a general face, and the integration by parts formula is

$$
\sum_{\Omega_{h}}(f, \alpha \cdot \nabla f)_{\Omega_{h}}=\frac{d}{2} \sum_{\Omega_{h}}\|f\|_{2, \Omega_{h}}^{2}+\frac{1}{2} \sum_{e_{i} \in E^{e}}\left\langle\left[f^{2}\right], \alpha \cdot \nu\right\rangle_{e_{i}}+\frac{1}{2}\left\langle f^{2}, \alpha \cdot \nu\right\rangle_{\partial \Omega} .
$$

For the estimates below, we need the following Lemma: 
LEMMA 3.2. Let $w_{h}, \psi \in \mathcal{V}_{P}$ be such that $w_{h}=\psi=0$ outside the bounded domain $\Omega$, and $V_{0}(x) \in L^{\infty}\left(\mathbb{R}^{d}\right)$. We have

$$
\left(\Theta\left[V_{0}\right]\left(w_{h}\right), \psi\right)_{\Omega} \leq c\left\|w_{h}\right\|_{2, \Omega}^{2}+\|\psi\|_{2, \Omega}^{2},
$$

where $c$ depends on $V_{0}$.

Proof. Since the potential $V_{0}$ is bounded, we have (see [22] and references therein)

$$
\begin{aligned}
\int_{\Omega} \Theta\left[V_{0}\right]\left(w_{h}\right) \psi d x d k & \leq\left\|\Theta\left[V_{0}\right]\left(w_{h}\right)\right\|_{L^{2}(\Omega)}^{2}+\|\psi\|_{L^{2}(\Omega)}^{2} \\
& \leq c\left\|V_{0}\right\|_{L^{\infty}\left(\mathbb{R}^{d}\right)}\left\|w_{h}\right\|_{L^{2}\left(\mathbb{R}^{2 d}\right)}+\|\psi\|_{L^{2}(\Omega)}^{2} \\
& \leq c\left\|w_{h}\right\|_{2, \Omega}^{2}+\|\psi\|_{2, \Omega}^{2}
\end{aligned}
$$

The consistency and stability of the scheme are proved in the following theorem:

THEOREM 3.1. Let $w_{h}(t)$ be the semi-discrete solution on $\mathcal{V}_{P}$ to (3.7). For all $T>0$ we have

$$
\begin{aligned}
& \left\|w_{h}(T)\right\|_{2, \Omega}^{2}+2 \sum_{\Omega_{h}} \int_{0}^{T}\left\|\nabla w_{h}\right\|_{2, \Omega_{h}}^{2} d t+2 \sum_{e_{i} \in E^{e}} \frac{1}{\left|e_{i}\right|} \int_{0}^{T}\left\|\left[w_{h}\right]\right\|_{2, e_{i}}^{2} d t \\
& +\sum_{e_{i} \in E^{e}} \int_{0}^{T}\left\|\left[w_{h}\right]|\alpha \cdot \nu|^{1 / 2}\right\|_{2, e_{i}}^{2} d t \leq\left\|w_{h}(0)\right\|_{2, \Omega}^{2} e^{d T}
\end{aligned}
$$

Proof. Use $\psi_{h}=w_{h}$ as the test function in (3.7). Using (3.9) we obtain

$$
\begin{aligned}
\frac{1}{2} \frac{d}{d t}\left\|w_{h}\right\|_{2, \Omega}^{2} & -\frac{d}{2} \sum_{\Omega_{h}}\left\|w_{h}\right\|_{2, \Omega_{h}}^{2}-\frac{1}{2} \sum_{e_{i} \in E^{e}}\left\langle\left[w_{h}^{2}\right], \alpha \cdot \nu\right\rangle_{e_{i}}+\sum_{e_{i} \in E^{e}}\left\langle w_{h}^{\uparrow}\left[w_{h}\right], \alpha \cdot \nu_{i}\right\rangle_{e_{i}} \\
& +\left(\Theta\left[V_{0}\right]\left(w_{h}\right), w_{h}\right)_{\Omega}+\sum_{\Omega_{h}}\left\|\nabla w_{h}\right\|_{2, \Omega_{h}}^{2}+\sum_{e_{i} \in E^{e}} \frac{1}{\left|e_{i}\right|}\left\|\left[w_{h}\right]\right\|_{2, e_{i}}^{2}=0 .
\end{aligned}
$$

The operator $\Theta\left[V_{0}\right]$ is skew-symmetric, which implies $\left(\Theta\left[V_{0}\right]\left(w_{h}\right), w_{h}\right)_{\Omega}=0$.

Identity (3.8) applied to the boundary terms on the interior edges leads to

$$
-\frac{1}{2} \sum_{e_{i} \in E^{e}}\left\langle\left[w_{h}^{2}\right], \alpha \cdot \nu\right\rangle_{e_{i}}+\sum_{e_{i} \in E^{e}}\left\langle w_{h}^{\uparrow}\left[w_{h}\right], \alpha \cdot \nu_{i}\right\rangle_{e_{i}}=\frac{1}{2} \sum_{e_{i} \in E^{e}}\left\langle\left[w_{h}\right]^{2},|\alpha \cdot \nu|\right\rangle_{e_{i}} .
$$

The identity above implies that

$$
\begin{aligned}
& \frac{d}{d t} \frac{1}{2}\left\|w_{h}\right\|_{2, \Omega}^{2}+\sum_{\Omega_{h}}\left\|\nabla w_{h}\right\|_{2, \Omega_{h}}^{2}+\sum_{e_{i} \in E^{e}} \frac{1}{\left|e_{i}\right|}\left\|\left[w_{h}\right]\right\|_{2, e_{i}}^{2} \\
& +\frac{1}{2} \sum_{e_{i} \in E^{e}}\left\|\left[w_{h}\right]|\alpha \cdot \nu|^{1 / 2}\right\|_{2, e_{i}}^{2} \leq \frac{d}{2}\left\|w_{h}\right\|_{2, \Omega}^{2} .
\end{aligned}
$$

The thesis follows from Gronwall's lemma. 
THEOREM 3.2. Let $w_{h}(t)$ be the semi-discrete solution in $\mathcal{V}_{P}$ to (3.7) for $t \geq 0$, and assume that $w_{I} \in H^{p+1}(\Omega), w(t) \in C^{\infty}(\Omega)$ for $t>0$. Then,

$$
\begin{aligned}
& \left\|\left(w-w_{h}\right)(T)\right\|_{2, \Omega}^{2}+\frac{1}{2} \sum_{\Omega_{h}} \int_{0}^{T}\left\|\nabla\left(w-w_{h}\right)\right\|_{2, \Omega_{h}}^{2} d t+\sum_{e_{i} \in E^{e}} \frac{1}{2\left|e_{i}\right|} \int_{0}^{T}\left\|\left[w-w_{h}\right]\right\|_{2, e_{i}}^{2} d t \\
& +\frac{1}{4} \sum_{e_{i} \in E^{e}} \int_{0}^{T}\left\|\left[w-w_{h}\right]|\alpha \cdot \nu|^{1 / 2}\right\|_{2, e_{i}}^{2} d t \\
\leq & c e^{T}\left(\left\|\left(w-w_{h}\right)(0)\right\|_{2, \Omega}^{2}+\mathbf{h}^{2 \mathbf{p}-\mathbf{2}} \int_{0}^{T}\left(\|w\|_{H^{p+1}(\Omega)}^{2}+\left\|\partial_{t} w\right\|_{H^{p}(\Omega)}^{2}\right) d t\right) .
\end{aligned}
$$

Proof. We consider the difference in the weak formulation for the functions $w$ and $w_{h}$, after decomposing the error $w-w_{h}$ into $w-w_{h}=\eta-\xi$ with $\eta:=w_{h}-w_{P}^{*}$ and $\xi=w-w_{P}^{*}$, where $w_{P}^{*}$ is the interpolant of $w$ in $\mathcal{V}_{P}$. We have that

$$
\begin{aligned}
& \left(\partial_{t} \eta, \psi\right)_{\Omega}-\sum_{\Omega_{h}}(\eta, \alpha \cdot \nabla \psi)_{\Omega_{h}}+\sum_{e_{i} \in E^{e}}\left\langle\eta^{\uparrow}[\psi], \alpha \cdot \nu_{i}\right\rangle_{e_{i}} \\
& +\frac{1}{2}\langle\eta \psi, \alpha \cdot \nu\rangle_{\partial \Omega}+\left(\Theta\left[V_{0}\right](\eta), \psi\right)_{\Omega} \\
= & \left(\partial_{t} \xi, \psi\right)_{\Omega}-\sum_{\Omega_{h}}(\xi, \alpha \cdot \nabla \psi)_{\Omega_{h}}+\sum_{e_{i} \in E^{e}}\left\langle\xi^{\uparrow}[\psi], \alpha \cdot \nu_{i}\right\rangle_{e_{i}}+\frac{1}{2}\langle\xi \psi, \alpha \cdot \nu\rangle_{\partial \Omega} \\
& +\left(\Theta\left[V_{0}\right](\xi), \psi\right)_{\Omega}-\sum_{\Omega_{h}}(\nabla \psi, \nabla \eta)_{\Omega_{h}}+\sum_{e_{i} \in E^{e}}\left\langle[\psi],\left\{\nabla \eta \cdot \nu_{i}\right\}\right\rangle_{e_{i}} \\
& -\sum_{e_{i} \in E^{e}}\left\langle[\eta],\left\{\nabla \psi \cdot \nu_{i}\right\}\right\rangle_{e_{i}}+\langle\psi, \nabla \eta \cdot \nu\rangle_{\partial \Omega}-\langle\eta, \nabla \psi \cdot \nu\rangle_{\partial \Omega} \\
& -\sum_{e_{i} \in E^{e}} \frac{1}{\left|e_{i}\right|}\langle[\eta],[\psi]\rangle_{e_{i}}+\sum_{\Omega_{h}}(\nabla \psi, \nabla \xi)_{\Omega_{h}}-\sum_{e_{i} \in E^{e}}\left\langle[\psi],\left\{\nabla \xi \cdot \nu_{i}\right\}\right\rangle_{e_{i}} \\
& +\sum_{e_{i} \in E^{e}}\left\langle[\xi],\left\{\nabla \psi \cdot \nu_{i}\right\}\right\rangle_{e_{i}}-\langle\psi, \nabla \xi \cdot \nu\rangle_{\partial \Omega}+\langle\xi, \nabla \psi \cdot \nu\rangle_{\partial \Omega} \\
& +\sum_{e_{i} \in E^{e}} \frac{1}{\left|e_{i}\right|}\langle[\xi],[\psi]\rangle_{e_{i}}=A_{1}+\ldots+A_{17} .
\end{aligned}
$$

We choose the test function $\psi=\eta$, and employ the same calculations as in the previous lemma. Also, due to the continuity of $\xi,[\xi]=0$, simplifying several terms,

$$
\begin{aligned}
A_{6} & =-\sum_{\Omega_{h}}\|\nabla \eta\|_{2, \Omega_{h}}^{2}, \quad A_{7}+A_{8}=0, \quad A_{9}+A_{10}=0, \\
A_{11} & =-\sum_{e_{i} \in E^{e}} \frac{1}{\left|e_{i}\right|}\|[\eta]\|_{2, e_{i}}^{2}, \quad A_{14}, A_{17}=0 .
\end{aligned}
$$

The combined result is,

$$
\begin{aligned}
& \frac{d}{d t}\|\eta\|_{2, \Omega}^{2}+\frac{1}{2} \sum_{e_{i} \in E^{e}}\left\|[\eta]|\alpha \cdot \nu|^{1 / 2}\right\|_{2, e_{i}}^{2}-\frac{d}{2}\|\eta\|_{2, \Omega_{h}}^{2} \\
& +\sum_{\Omega_{h}}\|\nabla \eta\|_{2, \Omega_{h}}^{2}+\sum_{e_{i} \in E^{e}} \frac{1}{\left|e_{i}\right|}\|[\eta]\|_{2, e_{i}}^{2}=A_{1}+\ldots+A_{5}+A_{12}+A_{13}+A_{15}+A_{16} .
\end{aligned}
$$


In the following estimates, we draw the reader's attention to the dependence on mesh size $h$ by using a bold faced $\mathbf{h}$.

Cauchy-Schwarz and the inverse inequality (3.3) produce the following estimates:

$$
\begin{aligned}
& A_{1} \leq c \mathbf{h}^{\mathbf{2} \mathbf{p}}\left\|\partial_{t} w\right\|_{H^{p}(\Omega)}^{2}+\|\eta\|_{2, \Omega}^{2} \\
& A_{2} \leq \frac{1}{h^{2}}\|\xi\|_{2, \Omega}^{2}\|\alpha\|_{\infty, \Omega}^{2}+\sum_{\Omega_{h}}\|\eta\|_{2, \Omega_{h}}^{2} \leq c_{|\Omega|} \mathbf{h}^{\mathbf{2} \mathbf{p}}\|w\|_{H^{p+1}(\Omega)}^{2}+\|\eta\|_{2, \Omega}^{2}
\end{aligned}
$$

Inequality (3.1) leads to:

$$
\begin{gathered}
A_{3} \leq \sum_{e_{i} \in E^{e}}\|\alpha\|_{\infty, e_{i}}\left\|\xi^{\uparrow}\right\|_{2, e_{i}}^{2}+\frac{1}{4} \sum_{e_{i} \in E^{e}}\left\|[\eta]\left|\alpha \cdot \nu_{i}\right|^{1 / 2}\right\|_{2, e_{i}}^{2}, \\
\leq c_{|\Omega|} \mathbf{h}^{\mathbf{2} \mathbf{p}+\mathbf{1}}\|w\|_{H^{p+1}(\Omega)}^{2}+\frac{1}{4} \sum_{e_{i} \in E^{e}}\left\|[\eta]\left|\alpha \cdot \nu_{i}\right|^{1 / 2}\right\|_{2, e_{i}}^{2}, \\
A_{4} \leq \frac{1}{2} h\|\eta\|_{2, \partial \Omega}^{2}+\frac{1}{2 h}\|\xi \alpha \cdot \nu\|_{2, \partial \Omega}^{2} \leq \frac{1}{2}\|\eta\|_{2, \Omega}^{2}+c_{|\Omega|} \mathbf{h}^{\mathbf{2} \mathbf{p}}\|w\|_{H^{p+1}(\Omega)}^{2} \\
A_{5} \leq\|\eta\|_{2, \Omega}^{2}+c \mathbf{h}^{\mathbf{2} \mathbf{p}+\mathbf{2}}\|w\|_{H^{p+1}(\Omega)}^{2}, \\
A_{12} \leq \frac{1}{4} \sum_{\Omega_{h}}\|\nabla \eta\|_{2, \Omega_{h}}^{2}+\sum_{\Omega_{h}}\|\nabla \xi\|_{2, \Omega_{h}}^{2}, \\
\leq \frac{1}{4} \sum_{\Omega_{h}}\|\nabla \eta\|_{2, \Omega_{h}}^{2}+c \mathbf{h}^{\mathbf{2} \mathbf{p}}\|w\|_{H^{p+1}(\Omega)}^{2}, \\
A_{13} \leq \sum_{e_{i} \in E^{e}}\left(\frac{1}{4 h}\|[\eta]\|_{2, e_{i}}^{2}+h\left\|\left\{\nabla \xi \cdot \nu_{i}\right\}\right\|_{2, e_{i}}^{2}\right) \\
\leq \frac{1}{4 h} \sum_{e_{i} \in E^{e}}\|[\eta]\|_{2, e_{i}}^{2}+c \mathbf{h}^{\mathbf{2} \mathbf{p}}\|w\|_{H^{p+1}(\Omega)}^{2} .
\end{gathered}
$$

Finally, using (3.2) gives,

$$
\begin{aligned}
A_{15} & \leq h\|\eta\|_{2, \partial \Omega}^{2}+\frac{1}{h}\|\nabla \xi \cdot \nu\|_{2, \partial \Omega}^{2} \\
& \leq c\|\eta\|_{2, \Omega}^{2}+c \mathbf{h}^{\mathbf{2} \mathbf{p}-\mathbf{2}}\|w\|_{H^{p+1}(\Omega)}^{2} \\
A_{16} & =\langle\xi, \nabla \eta \cdot \nu\rangle_{\partial \Omega} \leq \frac{1}{h}\left\|\xi^{2}\right\|_{2, \partial \Omega}+\frac{h}{4}\|\nabla \eta \cdot \nu\|_{2, \partial \Omega}^{2} \\
& \leq \mathbf{h}^{\mathbf{2} \mathbf{p}}\|w\|_{H^{p+1}(\Omega)}^{2}+\frac{1}{4}\|\nabla \eta\|_{2, \Omega}^{2} .
\end{aligned}
$$

Altogether, we have,

$$
\begin{aligned}
\frac{d}{d t}\|\eta\|_{2, \Omega}^{2} & +\frac{1}{4} \sum_{e_{i} \in E^{e}}\left\|[\eta]|\alpha \cdot \nu|^{1 / 2}\right\|_{2, e_{i}}^{2}+\frac{1}{4}\left\|[\eta]|\alpha \cdot \nu|^{1 / 2}\right\|_{2, \partial \Omega_{0}}^{2}+\frac{1}{2} \sum_{\Omega_{h}}\|\nabla \eta\|_{2, \Omega_{h}}^{2} \\
& +\sum_{e_{i} \in E^{e}} \frac{1}{2\left|e_{i}\right|}\|[\eta]\|_{2, e_{i}}^{2} \leq c\|\eta\|_{2, \Omega}^{2}+c_{|\Omega|} \mathbf{h}^{\mathbf{2 p}-\mathbf{2}}\left(\|w\|_{H^{p+1}(\Omega)}^{2}+\left\|\partial_{t} w\right\|_{H^{p}(\Omega)}^{2}\right) .
\end{aligned}
$$

The thesis then follows from Gronwall's lemma. 
3.3. On the spectrum of the WFP operator in the harmonic case. Some knowledge of the spectrum of the WFP operator provides a way to test the convergence rate of the DG method. When $V(x)=x^{2} / 2$ and $d=1$ equation (1.6) reads,

$$
w_{t}=x w_{k}-k w_{x}+w_{x x}+k w_{k}+w+w_{k k}=\mathcal{L} w, \quad(x, k) \in \mathbb{R}^{2} .
$$

The first eigenfunction of $\mathcal{L}$, with eigenvalue 0 , is $\mu$,

$$
\mu=(1 /(2 \sqrt{5} \pi)) \exp \left[-\left(x^{2} / 5+x k / 5+3 k^{2} / 10\right)\right] .
$$

Additional eigenfunctions can be produced by applying $\mathcal{L}$ to $P_{s}(x, k) \mu(x, k)$, where $P_{s}$ is an undetermined polynomial of degree $s$ in $x$ and $k$. The result is $Q_{s}(x, k) \mu(x, k)$, where $Q_{s}$ is a new polynomial. By solving the eigenvalue problem $P_{s}(x, k)=\lambda Q_{s}(x, k)$ for the unknown coefficients of $P_{s}$, one determines new eigenvalues and eigenfunctions of $\mathcal{L}$. Setting $s=1$ produces a conjugate pair of eigenvalues and eigenfunctions,

$$
\begin{aligned}
& \lambda_{ \pm 1}=-\frac{1}{2} \pm \frac{\sqrt{3}}{2} i \\
& \mu_{ \pm 1}=\left(\left(\frac{3}{14} \mp \frac{5 \sqrt{3}}{14} i\right) x+k\right) \mu(x, k) .
\end{aligned}
$$

We are only interested in real valued solutions of the WFP problem and consider the initial condition given by the sum

$$
\mu_{1}=\mu_{+1}+\mu_{-1}=\left(\frac{3}{7} x+2 k\right) \mu(x, k) .
$$

Note that $\mu_{1}$ has zero mass. If $w_{I}=\mu_{1}$, the solution to the WFP equation will converge to the trivial steady state, 0 , at the rate $\exp [-t / 2]$.

When $s=2$, three new eigenvalues are produced. One of the eigenvalues is real,

$$
\lambda_{2}=-1, \quad \mu_{2}=\left(x^{2}+x k+\frac{7}{3} k^{2}-\frac{20}{3}\right) \mu .
$$

The two remaining eigenvalues are a conjugate pair with real part also equal to -1 . Real combinations of all three eigenfunctions decay at rate $\exp [-t]$.

Another expected rate of convergence was described in equation (1.8). The weighted difference, $\|w-\mu\|_{L_{\mu}^{2}(\Omega)}$, will always decay at least as fast as $e^{-\sigma t}$, where $\sigma$ is the largest value such that $\operatorname{Hess}(A)-\sigma I \geq 0$. Numerical results exhibiting these convergence rates can be found in Section 4.1.

\section{Numerical Results}

The DG method described here has been implemented for $d=1$. The domain was partitioned into a regular rectangular mesh. The structure of the domain, $\Omega=\Omega^{x} \times \Omega^{k}$, makes this the natural choice, though in higher dimensions a more elaborate structure may be appropriate. To verify the numerical implementation, several tests were conducted using various potentials and different approximation spaces. In addition to confirming several known properties of the WFP equation, numerical tests were also performed which go beyond the scope of contemporary analysis.

For our numerical simulations we use the equation with the physical parameters, like in (1.2)-(1.4). We choose $\hbar=m=1$ and $\Omega=0$. 


\begin{tabular}{|c|c|c|c|c|}
\hline $\mathrm{N}$ & $L^{1}$ & $\begin{array}{c}\text { abs deviation } L^{1} \\
\text { slope }=-1.781\end{array}$ & $L_{\mu}^{2}$ & $\begin{array}{c}\text { abs deviation } L_{\mu}^{2} \\
\text { slope }=-1.698\end{array}$ \\
\hline 50 & $1.600 \mathrm{e}-02$ & $3.284 \mathrm{e}-03$ & $3.404 \mathrm{e}-02$ & $3.894 \mathrm{e}-03$ \\
72 & $8.480 \mathrm{e}-03$ & $3.079 \mathrm{e}-03$ & $1.865 \mathrm{e}-02$ & $3.616 \mathrm{e}-03$ \\
94 & $5.276 \mathrm{e}-03$ & $3.247 \mathrm{e}-03$ & $1.187 \mathrm{e}-02$ & $3.867 \mathrm{e}-03$ \\
116 & $3.605 \mathrm{e}-03$ & $0.499 \mathrm{e}-03$ & $8.242 \mathrm{e}-03$ & $0.663 \mathrm{e}-03$ \\
138 & $2.621 \mathrm{e}-03$ & $3.535 \mathrm{e}-03$ & $6.068 \mathrm{e}-03$ & $4.255 \mathrm{e}-03$ \\
\hline
\end{tabular}

TABLE 4.1. Convergence to the analytic steady state of the harmonic potential with respect to mesh refinement. A regular $N \times N$ grid was used in each case. The log of $\left\|\mu_{h}-\mu\right\|_{L^{1}(\Omega)}$ and $\left\|\mu_{h}-\mu\right\|_{L_{\mu}^{2}(\Omega)}$ are reported in columns two and four. These values decrease linearly with the log of $N$. The deviation between each datum and a best fit linear relationship is reported in columns three and five under "abs deviation." The slope of the best fit trend is reported in the respective column header.

4.1. Verification. The first and most basic tests showed that a number of subproblems contained within the WFP equation are correctly solved. Two of the tests check for convergence of the numerical solution to the time dependent solutions of the transport and heat equations with respect to mesh and basis set order. These tests were successful, achieving the expected convergence and rates.

Those initial tests were trivial in that they did not involve the pseudo-differential operator. To test the implementation of $\Theta[V]$, we verified that simulations converge at the proper rate to the known stationary state of the WFP problem using a harmonic potential, $V(x)=x^{2} / 2$. The tests in this Section (4.1) were carried out under the following conditions: all constants were set equal to unity, as in equation (1.6); the computational domain and time-step used were $\Omega=[-10,10] \times[-10,10]$ and $d t=0.001$ respectively; in order to measure the rate of convergence to the steady state, the initial state is a Gaussian function, normalized and centered at the origin,

$$
w_{I}=(2 / \pi) \exp \left[-2\left(x^{2}+k^{2}\right)\right] .
$$

The amount of mass not contained in $\Omega$ is approximately $7.8 \times 10^{-9}$ for $\mu$, and less than $10^{-10}$ for $w_{I}$. These values are much smaller than other sources of numerical error (see the introduction for a discussion of the consequences of working in a cut-off domain).

The numerical implementation uses one of three different approaches to evaluate the pseudo-differential operator depending on the form of the potential. The first method examined was the "classical" approach, that replaces the pseudo-differential with (1.5) because the potential is harmonic.

Table 4.1 shows convergence to (3.10) with respect to mesh refinement. The simulations were carried out using $\mathcal{V}_{\mathcal{P}}^{1}$, piecewise linear functions. Each simulation was evolved until a numerical steady state, $\mu_{h}$, was (approximately) reached. The table lists $L^{1}(\Omega), L_{\mu}^{2}(\Omega)$, and corresponding "abs deviation" values. The "abs deviation" columns indicate the absolute value of the difference between the data in columns two and four and a best fit linear relationship between the functions $\log (N)$ and $\log ($ err $)$. The slope of these linear relationships is listed at the top of each column. The small values in these columns indicate linear behavior, that is, exponential convergence to the analytic steady state as the mesh is refined.

In the case of the harmonic potential, the steady state is unique, so we repeated some of the calculations above with different initial data. One example is a combina- 


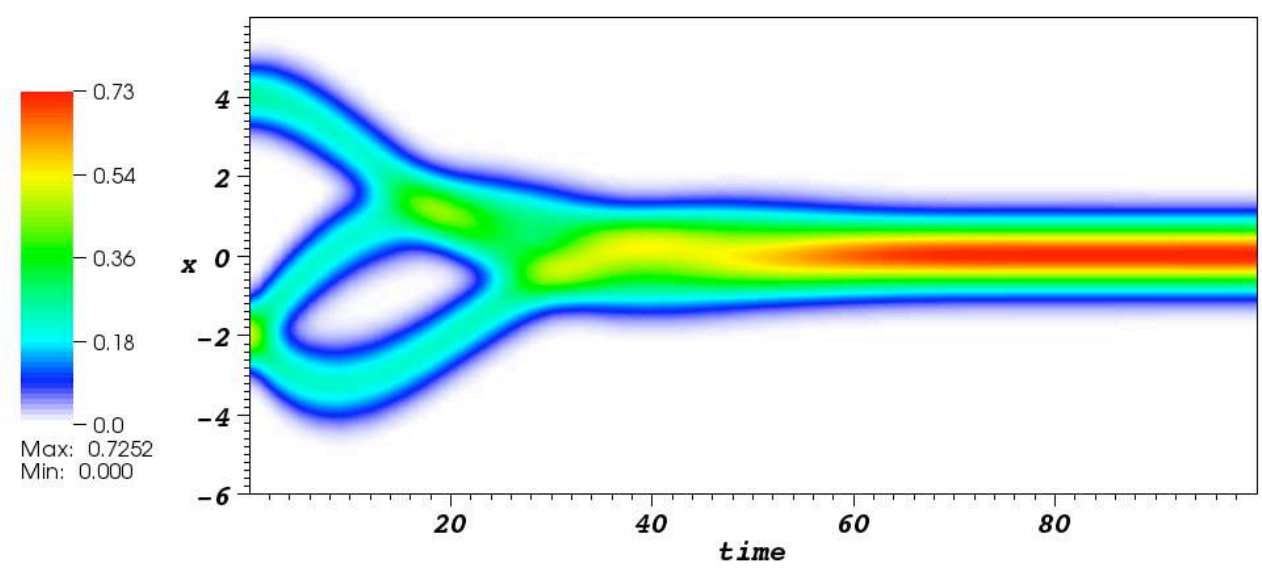

Figure 4.1. Density plot of the convergence of a three centered initial state to the unique steady state of WFP equation with a harmonic potential. The numerical solution is essentially zero in the white regions.

tion of three Gaussians centered about the origin,

$$
\begin{aligned}
w_{I}=\frac{2}{3 \pi} & \left(\exp \left[-2\left((x-4)^{2}+(k-0)^{2}\right)\right]+\exp \left[-2\left((x+2)^{2}+(k-2 \sqrt{3})^{2}\right)\right]\right. \\
& \left.+\exp \left[-2\left((x+2)^{2}+(k+2 \sqrt{3})^{2}\right)\right]\right) .
\end{aligned}
$$

Convergence is depicted in figure 4.1, a plot of charge density, $\rho(x, t)=w(x, k, t) d k$, as a function of $x$ and $t$. Since density is a projection of the solution onto $x$ and $t, w_{I}$ initially appears to have only two centers due to a symmetry, which is immediately broken as the three centers spiral around the origin. The steady state achieved in this calculation is $\mu$, which is shown in figure 4.2 (using a $64 \times 64$ grid), labelled "three Gaussians". The rate of convergence to $\mu$ is controlled by the spectrum of the WFP operator. Some of its eigenvalues were derived, for the harmonic case, in section 3.3, and a bound on these values, is known: $-\sigma \approx-0.276$. figure 4.2 is a plot of $\log \left\|w_{h}-\mu\right\|_{L_{\mu_{h}}^{2}(\Omega)}$ as a function of time for several different initial conditions. The grid used is $64 \times 64$, the approximation space is $\mathcal{V}_{\mathcal{P}}^{1}$, and the remaining parameters are those stated at the beginning of this section. Both panels show the same data, but the first is plotted on a shorter time-scale to highlight the initial convergence behavior. The rate is calculated with respect to the numerical steady state, $\mu_{h}$, as determined by the calculation. If the analytic steady state is used instead, approximation error obscures the long term behavior. Convergence rate is the primary object of interest, so each curve in 4.2 has been translated to intersect the origin. Results are shown for five different initial conditions: "asymmetric," $w_{I}=x \mu+\mu$, which initially decays at the slowest possible rate, $-\sigma$; "first eigenfunction," $\mu_{1}+\mu$, from equation (3.11), which initially decays with rate $-1 / 2$; "second eigenfunction," $\mu_{2}+\mu$, from equation (3.12), which initially decays at rate -1 ; "one Gaussian," equation (4.1); and "three Gaussians," equation (4.2). The steady state $\mu$ was added to the first and second eigenfunctions so that the solution would have unit mass and therefore converge to $\mu$ rather than the trivial steady state, 0 , which cannot be used to produce the weighted 
$L^{2}$ norm. The traces in the figure oscillate, but reveal several distinct slopes, revealing information about the spectrum of the WFP operator. To further test the "classical"
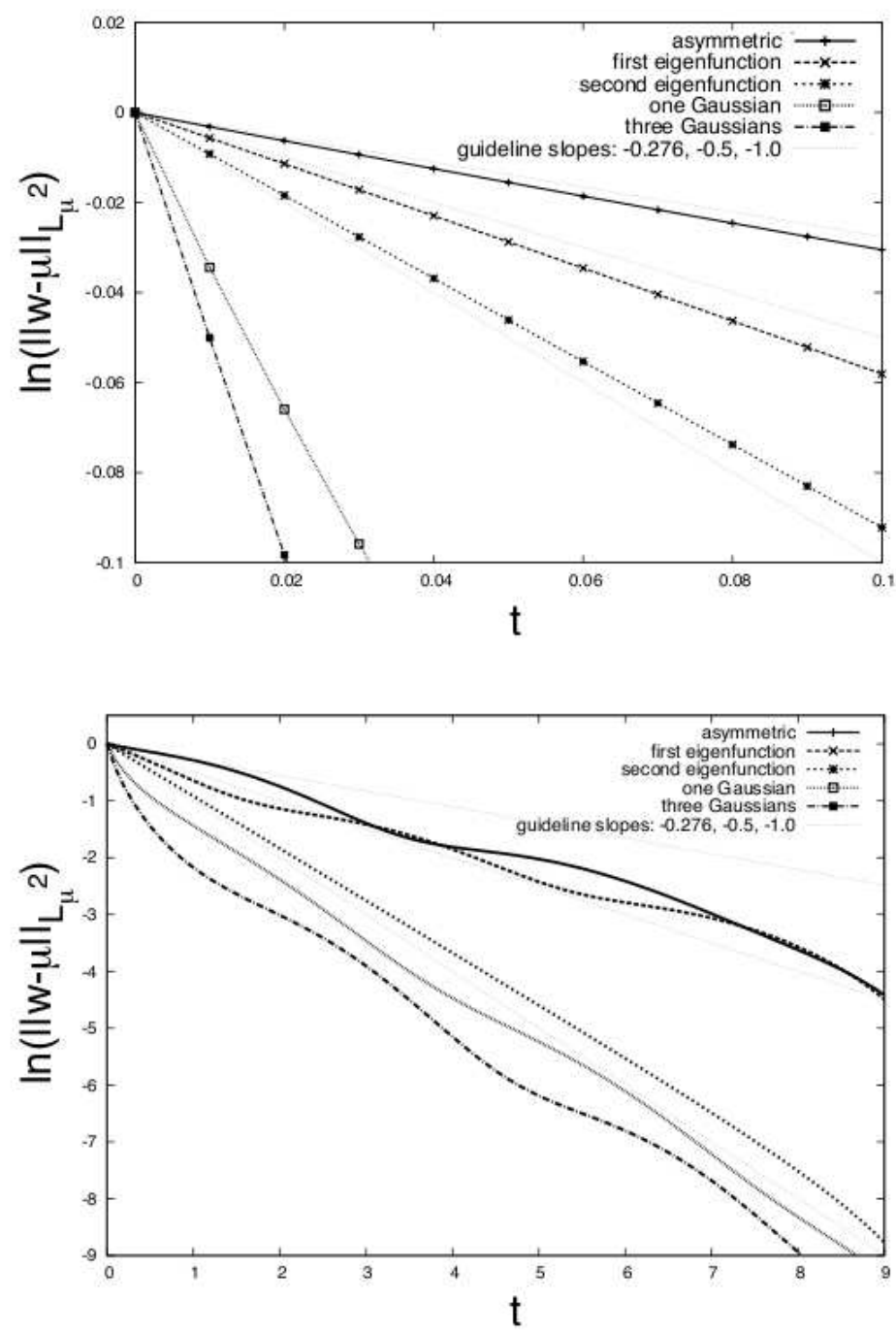

FIGURE 4.2. The $L_{\mu_{h}}^{2}$ convergence of the solution to the unique steady state (equation 3.10) of the harmonic potential. The two figures show the same data on different time scales. The figure on the left highlights the initial decay rates using various initial conditions, as described in the text. To simplify comparison between curves, they have all been shifted to intersect the origin. The rate of convergence can be no slower than $\exp (-\sigma t)$ where $\sigma=(1-1 / \sqrt{5}) / 2 \approx 0.276$. Lines with slopes $-1 / 2,-1$, and $-\sigma$ are drawn, corresponding to two eigenvalues of the WFP operator and the bound on the decay rate.

implementation of the pseudo-differential operator, several runs were performed using each of the different approximation spaces. The same parameters were used, but the mesh size and approximation space were varied. The results are listed in Table 4.2. The variable $m$ is the number of primitive basis functions used to construct 
the approximation space on each cell in the mesh, consequently, there are $m^{2}$ basis functions on each cell. For the polynomial and Hermite bases, $m$ is equal to $p+1$. For the trigonometric case, the sets of primitive basis functions for $m=1,2$, and 3 are $\{1\},\{1, \cos \}$, and $\{1, \cos , \sin \}$ respectively (no $m=4$ trigonometric approximation space was implemented). The period of each primitive trigonometric basis function was equal to the width of its cell, in the respective coordinate, $x$ or $k$. The restricted Hermite approximation spaces have more structure, and they are described in detail below.

The purpose of these tests was to show the advantages and disadvantages of various approximation spaces, so the meshes are relatively coarse in order to magnify differences. The polynomial basis is in fact optimal as $N$ becomes large, but sub-grid resolution can be exploited on coarse meshes to boost efficiency. As a consequence of equation (3.7), mass is conserved when the approximation space is piecewise constant. This was observed, up to machine precision, and furthermore, in all polynomial and trigonometric cases, the total mass was preserved to at least $0.1 \%$. However, the Hermite approximation space does not include piecewise constant solutions. Hermite functions are products of Hermite polynomials and a Gaussian function. In these cases conservation of mass was obtained through the following procedure: a parameter controlling the width of the Gaussian part of the Hermite functions was adjusted until mass was conserved to better than $0.1 \%$ after 10,000 timesteps. This parameter is very similar to the $\alpha_{j}$, which appear in equation (3.1) of [28]. Shu has indicated to the authors that there is not a general approach for selecting this parameter in such a way as to preserve mass. That is, the approximation space was optimized by hand to give good subgrid resolution on very coarse meshes.

Column one shows the error, $\left\|\mu_{h}-\mu\right\|_{L^{1}(\Omega)}$, and column two shows the log (base 10 ) of the weighted $L_{\mu}^{2}$ norm. Essentially, column one gives the absolute error, and column two measures the quality of the solution in a relative sense. There is a clear trend of improvement as the mesh and basis set are refined for the polynomial approximation spaces, with one exception. In the piecewise constant case, the $N=16$ grid is best. A piecewise constant basis is actually unsuitable for this problem, for example, because the contribution from the second order terms in the Fokker-Planck operator are eliminated, and the solution fails to converge to $\mu$, becoming tightly peaked around the origin. The trigonometric approximation spaces also fare poorly. Qualitatively, they produce a peak which is too flat and broad.

Hermite functions are the eigenfunctions of the quantized Hamiltonian with a harmonic potential (see for example [9]), and are very similar to the eigenfunctions of the WFP equation (see the discussion of figure 4.2). That is, the approximation space is made of functions very similar to the desired solution. The result is a good solution even on the $N=2$ mesh. As more Hermite functions are added and the mesh becomes coarser, the method is nearly a spectral solver in a cut-off domain.

4.2. Calculations for perturbations of the harmonic potential. The next challenge is to verify the implementation of the convolution based methods for evaluating the pseudo-differential operator acting on the perturbation potential, where the only (non-trivial) analytic solution is for the harmonic potential. There is already a good approach for harmonic potentials, and because the inverse Fourier transform of the harmonic potential is a distribution, it is not possible to simply insert it into the convolution based numerical calculations used to evaluate $\Theta[V](w)$. However, the harmonic potential can be approximated locally, i.e., everywhere within the cut-off domain, by a simple combination of sinusoidal functions. In all subsequent calcula- 


\begin{tabular}{|c|c|c|c|c|}
\hline \multicolumn{5}{|c|}{$\left\|\mu_{h}-\mu\right\|_{L^{1}}$} \\
\hline $\mathcal{V}_{\mathcal{P}}^{p}$ & $\mathrm{~m}$ \\
\hline $\mathrm{N}$ & 1 & 2 & 3 & 4 \\
\hline 2 & & & & \\
4 & 1.485 & 0.484 & 0.278 & 0.198 \\
8 & 0.970 & 0.178 & 0.109 & 0.023 \\
16 & 0.356 & 0.106 & 0.044 & 0.003 \\
32 & 0.812 & 0.035 & 0.018 & 0.000 \\
\hline
\end{tabular}

\begin{tabular}{|c|c|c|c|c|}
\hline \multicolumn{5}{|c|}{$\log _{10}\left(\left\|\mu_{h}-\mu\right\|_{L_{\mu}^{2}}\right)$} \\
\hline $\mathcal{V}_{\mathcal{P}}^{p}$ & $\mathrm{~m}$ & & \\
\hline $\mathrm{N}$ & 1 & 2 & 3 & 4 \\
\hline 2 & & & & \\
4 & 11.866 & 10.357 & 9.002 & 8.547 \\
8 & 10.435 & 6.465 & 4.536 & 3.029 \\
16 & 7.125 & 0.939 & -0.927 & -2.186 \\
32 & 0.275 & -1.140 & -1.550 & -3.523 \\
\hline
\end{tabular}

\begin{tabular}{|c|c|c|c|c|}
\hline $\mathcal{V}_{T}$ & \multicolumn{1}{|c|}{$\mathrm{m}$} & \multicolumn{3}{l|}{} \\
\hline $\mathrm{N}$ & 1 & 2 & 3 & 4 \\
\hline 2 & & & & \\
4 & 1.486 & 1.920 & 1.318 & \\
8 & 0.970 & 1.263 & 1.102 & \\
16 & 0.356 & 0.419 & 0.916 & \\
32 & 0.812 & 0.772 & 0.748 & \\
\hline
\end{tabular}

\begin{tabular}{|c|c|c|c|c|}
\hline $\mathcal{V}_{T}$ & \multicolumn{1}{|c|}{$\mathrm{m}$} & \multicolumn{3}{l|}{} \\
\hline $\mathrm{N}$ & 1 & 2 & 3 & 4 \\
\hline 2 & & & & \\
4 & 11.866 & 11.344 & 11.609 & \\
8 & 10.435 & 11.625 & 10.572 & \\
16 & 7.125 & 9.433 & 8.529 & \\
32 & 0.275 & 3.166 & 6.212 & \\
\hline
\end{tabular}

\begin{tabular}{|c|c|c|c|c|}
\hline $\mathcal{V}_{H}^{p}$ & \multicolumn{2}{|c|}{$\mathrm{m}$} & \multicolumn{3}{|c|}{} \\
\hline $\mathrm{N}$ & 1 & 2 & 3 & 4 \\
\hline 2 & 0.321 & 0.124 & & \\
4 & 0.320 & & & \\
8 & & & & \\
16 & & & & \\
32 & & & & \\
\hline
\end{tabular}

\begin{tabular}{|c|c|c|c|c|}
\hline $\mathcal{V}_{H}^{p}$ & \multicolumn{2}{|c|}{$\mathrm{m}$} & \multicolumn{2}{|c|}{} \\
\hline $\mathrm{N}$ & 1 & 2 & 3 & 4 \\
\hline 2 & -0.301 & -0.470 & & \\
4 & -0.303 & & & \\
8 & & & & \\
16 & & & & \\
32 & & & & \\
\hline
\end{tabular}

TABLE 4.2. A calculation on relatively coarse grids shows the effect of various approximations spaces. The left column reports the $L^{1}$ error between the numerical and analytic steady states (absolute error), and right column gives the logarithm (base 10) of the weighted $L^{2}$ error (roughly, the relative error). The value $m$ is the number of primitive basis functions used to construct each approximation space.

tions the basis used is $\mathcal{V}_{\mathcal{P}}^{1}$, the piecewise linear polynomial basis. We stress that we have found this is the most efficient one to use for finer meshes.

4.2.1. Small sinusoidal perturbations. The method for evaluating $\Theta[V](w)$, where the potential consists of sinusoidal functions, (2.10), was therefore tested by showing that a sequence of non-harmonic potentials converging locally to $V(x)=x^{2} / 2$ produces a sequence of stationary states which converge to the stationary state of the related harmonic problem. The sequence of potentials, parameterized by $a_{i}$, is,

$$
\begin{aligned}
V\left(x ; a_{i}\right) & =a_{i}\left(1-\cos \left(\frac{x}{\sqrt{a_{i}}}\right)\right) \\
& =\frac{1}{2} x^{2}+\mathcal{O}\left(\frac{x^{4}}{a_{i}^{2}}\right) .
\end{aligned}
$$

The parameters used were the same as those used to produce Table 4.1. A $94 \times 94$ mesh was used. The calculation was run to a steady state, and this steady state was compared to the same numerical steady state which was used to produce the third row of Table 4.1. The result demonstrating the desired convergence appears in Table 


\begin{tabular}{|r|c|c|}
\hline \multicolumn{1}{|c|}{$a_{i}$} & $\left\|\mu_{a_{i}}-\mu_{h}\right\|_{L^{1}(\Omega)}$ & $\left\|\mu_{a_{i}}-\mu_{h}\right\|_{L_{\mu_{h}}^{2}(\Omega)}$ \\
\hline 10.0 & $6.019 \mathrm{e}-02$ & $7.024 \mathrm{e}-01$ \\
20.0 & $2.776 \mathrm{e}-02$ & $6.967 \mathrm{e}-02$ \\
30.0 & $1.824 \mathrm{e}-02$ & $4.173 \mathrm{e}-02$ \\
50.0 & $1.094 \mathrm{e}-02$ & $2.369 \mathrm{e}-02$ \\
100.0 & $5.588 \mathrm{e}-03$ & $1.150 \mathrm{e}-02$ \\
\hline
\end{tabular}

TABLE 4.3. Convergence to the harmonic potential steady state for the potential, $V\left(x ; a_{i}\right)=$ $a_{i}\left(1-\cos \left(x / \sqrt{a_{i}}\right)\right)$. As the parameter $a_{i}$ increases, the potential converges locally to $x^{2} / 2$, and the numerical steady state, $\mu_{a_{i}}$, converges to the numerical steady state of the purely harmonic problem, $\mu_{h}$.

4.3 .

4.2.2. Particle in a box. The final method for evaluating the pseudodifferential operator which needs to be tested is the explicit convolution method, (2.9). This test was qualitative, and similar to the well known "particle in a box" problem. Again, it consisted of setting $w_{I}$ equal to a Gaussian centered at the origin and evolving the solution. A combination of Heaviside functions was used to create a set of four potential barriers with various heights and widths. The barriers were placed on the intervals $[-11,-10],[-4.1,-4],[4,4.1]$, and $[10,11]$. The height of the outer barriers is ten times greater than the inner barriers. The outer barriers are essentially impenetrable, and meant to confine the solution. The inner barriers restrict, but do not completely contain $w_{h}$ as it evolves. The diffusion constants were $D_{q q}=D_{p p}=0.1$. The domain was $\Omega=[-15,15] \times[-4,4]$ and was divided into a $128 \times 64$ mesh. The time-step was $d t=0.001$. The solution, after some 50,000 timesteps, is pictured in figure 4.3. It has not reached a steady state, but rather is slowly leaking from the center box into the outer boxes. At this point, due to the relatively long time-step, it becomes difficult to determine whether the solution is changing due to numerical mass loss or a slow physical process. The solution has clearly been contained by the barriers in a qualitatively appropriate manner.

4.2.3. Triple well. Finally, the method was used to calculate the behavior of an initial Gaussian in a perturbed confining potential. The potential used was,

$$
V(x)=\frac{x^{2}}{2}+30(1-\cos (x)) .
$$

The potential is pictured in the inset in figure 4.4 and has three deep wells, at the origin and near $\pm 2 \pi$. In this case, the initial state was a Gaussian concentrated about a large positive value of $x$ and negative value of $k$ to give it a rapid initial motion toward the origin. A projection of the initial state onto the $x$ axis is also provided in the inset to figure 4.4 (its width has been exaggerated for the purpose of illustration). During the course of the simulation, the center of mass (charge) flows down the potential wall, and a short way up the other side before becoming concentrated in two of the wells. The main panel in figure 4.4 shows a plot of charge density in the $x, t$ plane (it is analogous to figure 4.1). The total mass is preserved to within $10^{-5}$ during the simulation. The time-step was $d t=0.001, \Omega=[-20,30] \times[-35,15]$, and the mesh measured $200 \times 200$. At any given time, the total mass contained outside the 0.005 contour level (the white area) is essentially zero. The vertical lines at $t=33$, 37,41 , and 45 correspond the snapshots of the solution shown in figure 4.5. The 


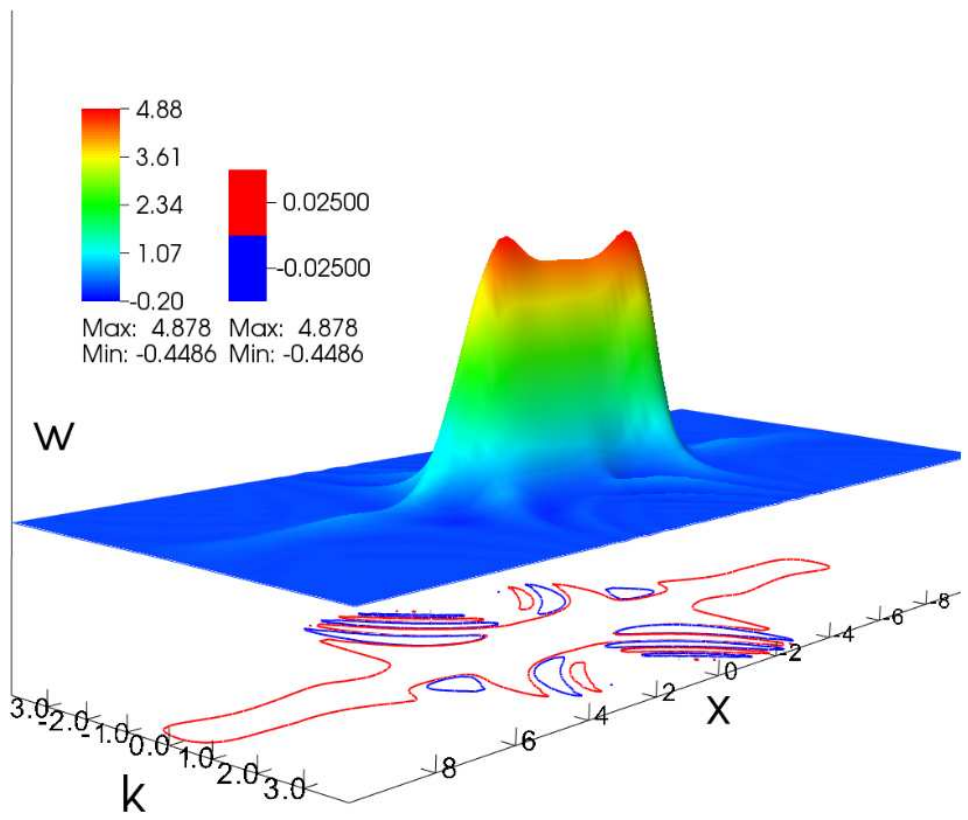

(a)

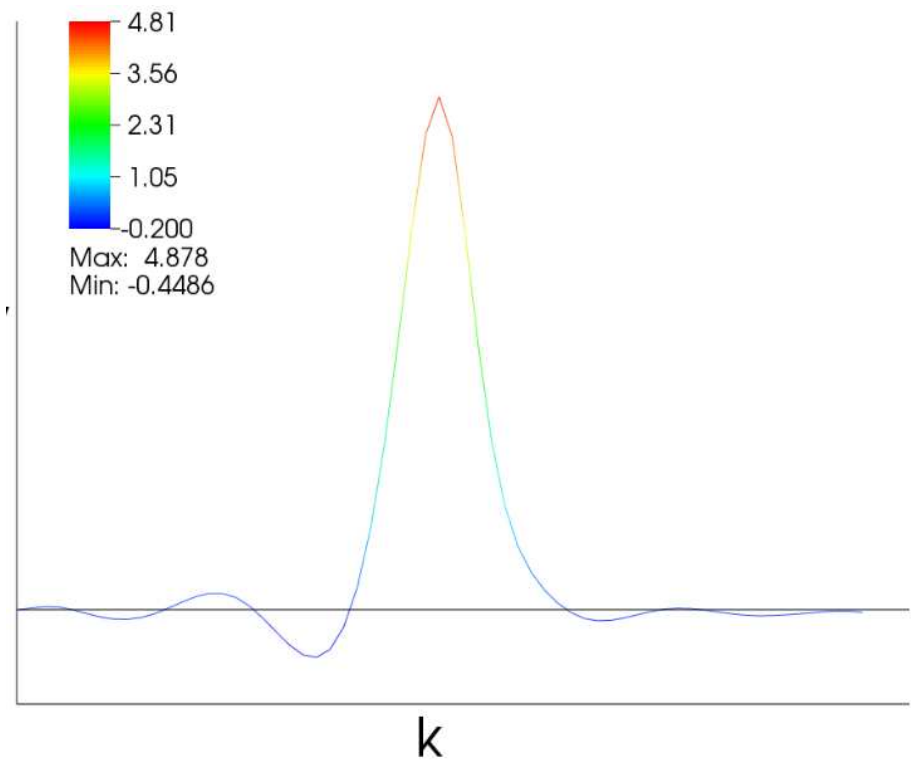

(b) $x=2$

FIGURE 4.3. The numerical solution to the (quasi) "particle in a box" problem after 50,000 timesteps. The potential consists of low barriers placed at $x= \pm 4$ and high barriers at $x= \pm 10$. Most, but not all of the solution is contained by the low barriers. When the calculation was stopped, the solution was leaking slowly from the central bin $[-4,4]$ into the adjacent bins $[-10,-4.1]$ and $[4.1,10]$. 


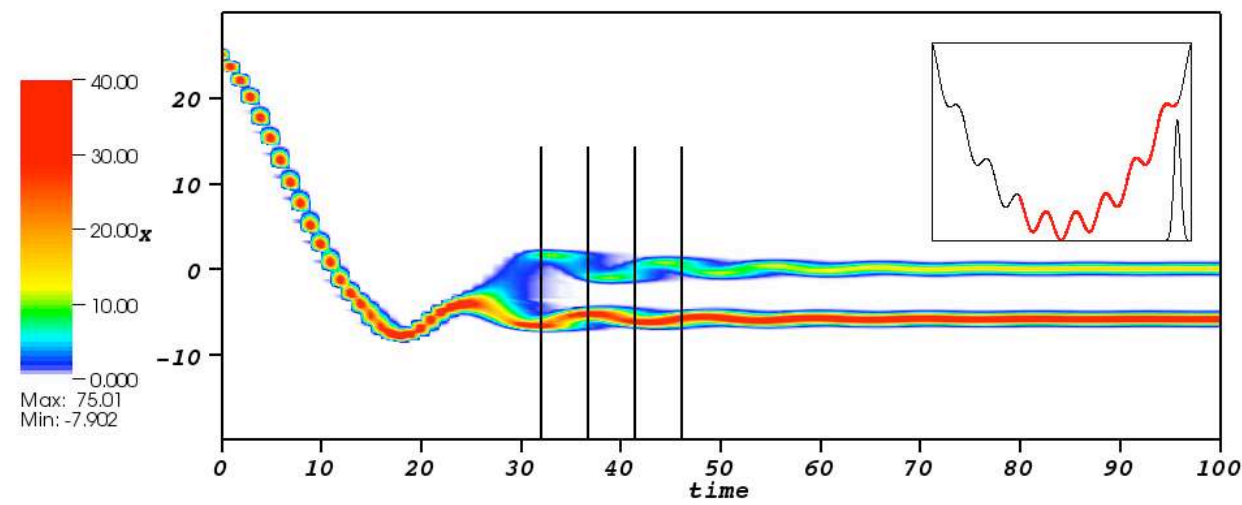

FIGURE 4.4. A plot of charge density in the $x, t$ plane. The solution in the white area is negligible. The inset shows the potential, and a projection of the initial state (the width has been exaggerated for illustration). The bold part of the potential indicates the locations of the center of mass during the calculation. The solution eventually settles into two of the three deep wells of this potential at $x=0$ and $x \approx-2 \pi$. The four vertical lines indicate the points in time which were used to create the frames in figure 4.5 .

frame at $t=33$ represents a moment when the solution is spilling over from the well at $x \approx-2 \pi$ into the well at the origin. The remaining frames show each part of the solution completing a circuit of its respective well. As indicated by figure 4.4, the solution settles into these wells and in each case takes on an appearance very similar to a pair of Gaussians.

A close examination shows that at least a small but measurable portion of the solution may be found in four wells, the three central wells, and the well close to $4 \pi$. A contour map of the solution, with contour levels close to zero, is shown in figure 4.6. Numbers appear at the center of each well along the $x$ axis. Also, three pairs of horizontal lines indicate the minimum amount of classical kinetic energy required to escape each of the marked wells. That is, at values of $k$ outside of the innermost pair of lines, there is enough kinetic energy to overcome the potential barrier between wells 4 and 3 . The center set of lines indicates the energy needed to move from either well 1 or 3 into well 2. The outermost pair of lines is the amount of energy needed to accomplish the reverse, escaping well 2 . The vast majority of the solution is contained within wells 1 and 2 . The mass contained inside the $10^{-3}$ contour is approximately 0.991 (out of 1.000). The mass contained outside of the $10^{-6}$ contour is only equal to about $1.57 \times 10^{-5}$. While it is doubtful that the solution has reached a stationary state, it is clear that it has settled into a persistent asymmetric configuration. By the symmetry in the equation itself, given initial data which is symmetric with respect to this potential, the solution will remain symmetric. This was confirmed by further simulations. Given that a stationary state to this linear problem does indeed exist, the asymmetric result of the calculation is most likely a metastable state, and that the relaxation of this state to a symmetric steady state occurs on a timescale much longer than the interval covered by the calculation. A very recent work by Arnold, Fagnola, and Neumann on existence, uniqueness, and convergence in the case of sub-quadratic perturbations to the harmonic potential supports this observation [5]. 


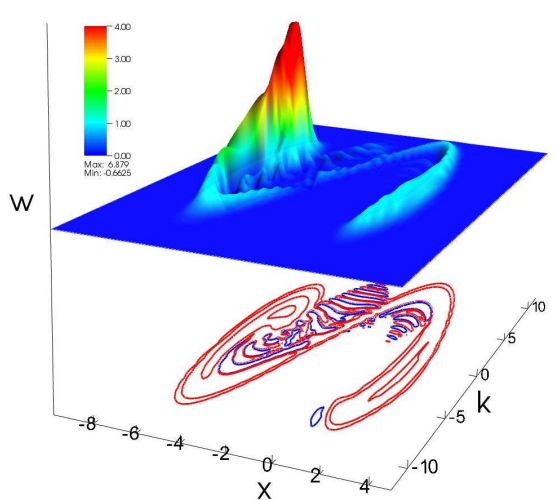

(a) $t=33$

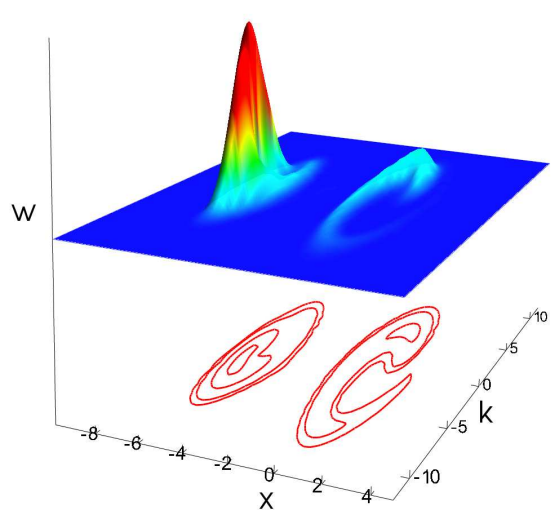

(c) $\mathrm{t}=41$

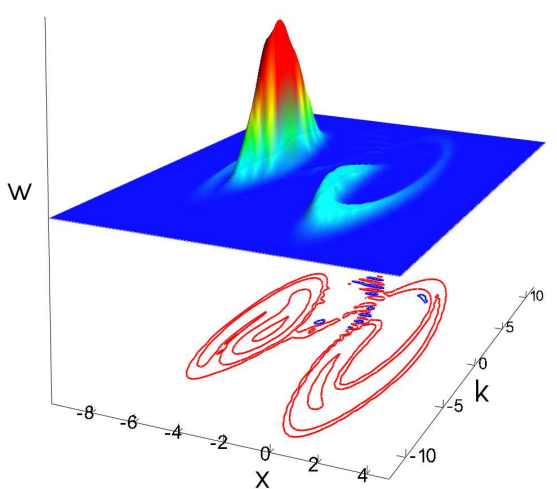

(b) $\mathrm{t}=37$

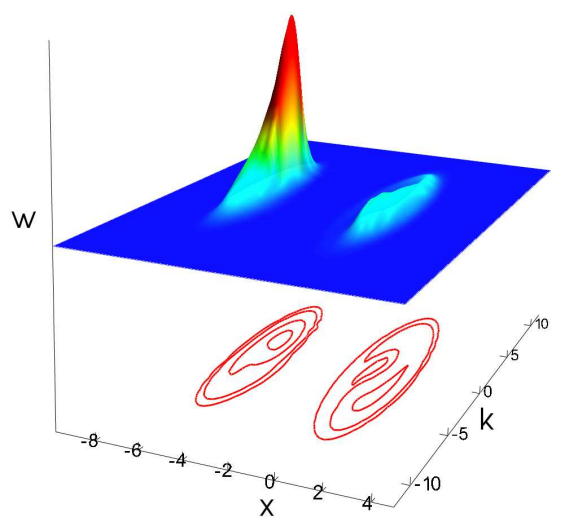

(d) $\mathrm{t}=45$

FIGURE 4.5. Snapshots of the solution to the triple well problem. These figures show the solution completing a circuit of the wells located at $x \approx-2 \pi$ and $x=0$. They correspond to the vertical lines in figure 4.4.

\section{Conclusion}

The utility of the DG method developed here for the WFP equation has been demonstrated through analytic estimates and numerical experiments. The stability and convergence of the scheme were established in Section 3, and numerical simulation was used to confirm analytic properties of the WFP equation in section 4 . The main challenge was to produce an accurate and practical treatment of the pseudo-differential term. The methods described in section 2 do this in a manner that falls neatly into the DG formalism, and a wide range of potential functions may be treated. Linear combinations of harmonic, sinusoidal, and step functions were demonstrated, and it is clear how to apply the method to Gaussian and other families of potential functions.

The numerical simulations presented here demonstrated that the use of non- 


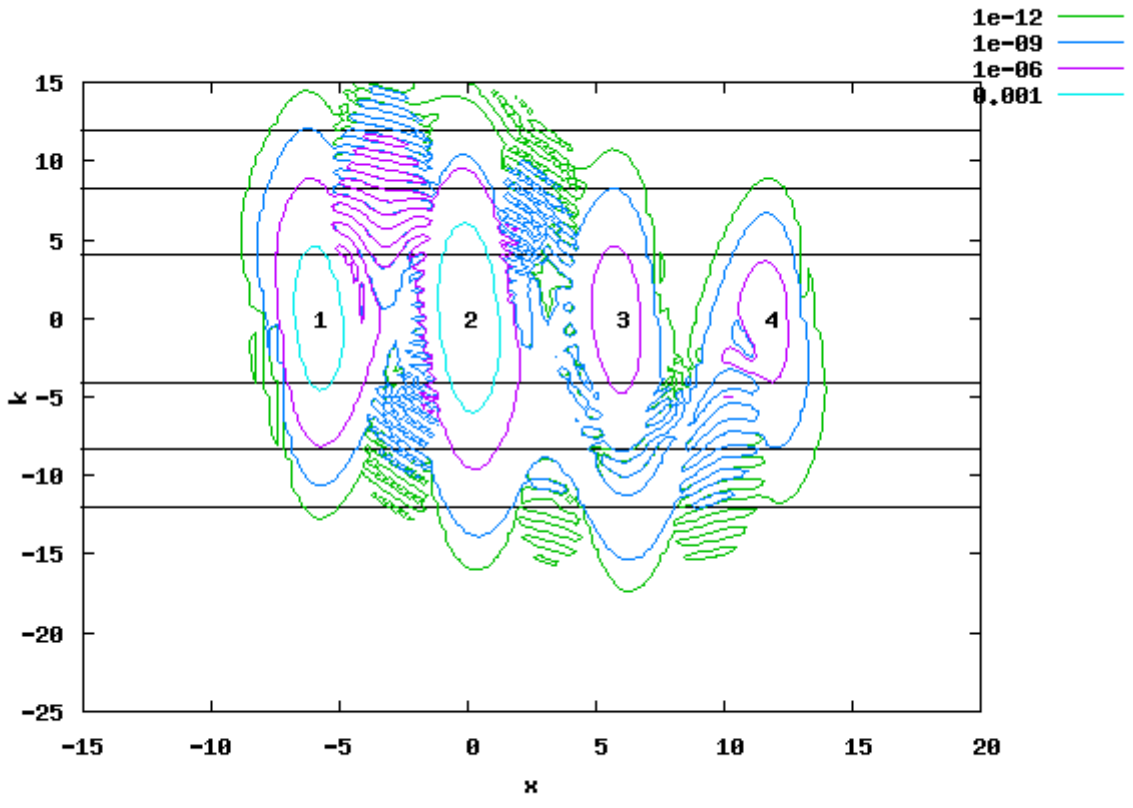

FIGURE 4.6. A contour map of the state eventually reached in the triple-well calculation. The solution is positive, and the contour levels are set close to zero. More than $90 \%$ of the mass lies within the 0.001 contour, that is, within wells 1 and 2 . Other contours reveal small concentrations of the solution in two additional wells. Pairs of horizontal lines, symmetric about $k=0$ indicate the amount of classical kinetic energy needed to overcome the energy barriers from well 4 to 3 (the innermost pair), either well 1 or 3 to well 2 (the central pair), or to escape well 2 (the outermost pair).

polynomial approximation spaces is possible, but did not extensively explore the possibilities offered by this flexible framework. A possible improvement to the method, also suggested in the work by Yuan and Shu, will be to adaptively improve the approximation space as a computation proceeds. Other proposed technical improvements to the implementation include expansion to three dimensions, and coupling the WFP with the Poisson equation. One approach might be to use an FFT solver for the Poisson problem, which would produce a sinusoidal representation of the potential ready for use in the method reported here.

Acknowledgement. We would like to thank Jennifer Proft and Anton Arnold for several helpful discussions. I. M. Gamba was partially supported under the NSF grant DMS-0507038. M. P. Gualdani was partially supported under the Feodor Lynen Research fellowship and NSF grant DMS-0807636. Support from the Institute of Computational Engineering and Sciences and the University of Texas Austin is also gratefully acknowledged. 


\section{REFERENCES}

[1] A. Arnold, Self-consistent relaxation-time models in quantum mechanics, Commun. Part. Differ. Equs., 21, 473-506, 1996.

[2] A. Arnold, J.A. Carillo and E. Dhamo, On the periodic Wigner-Poisson-Fokker-Planck system, J. Math. Anal. Appl., 275, 263-276, 2002.

[3] A. Arnold, E. Dhamo and C. Manzini, Dispersive effects in quantum kinetic equations, Indiana University Mathematics Journal, 56, 1299-1332, 2007.

[4] A. Arnold, E. Dhamo and C. Manzini, The Wigner-Poisson-Fokker-Planck system: global-intime solutions and dispersive effects, Annales de l'Institut Henri Poincaré (C) Analysis non Linéaire, 24, 645-676, 2007.

[5] A. Arnold, F. Fagnola and L. Neumann, Quantum Fokker-Planck models: the Lindblad and Wigner approaches, in Quantum Probability and Related Topics - Proceedings of the 28th Conference, J.C. García, R. Quezada, and S. B. Sontz (eds.), QP-PQ: Quantum Probability and White Noise Analysis, World Scientific, 23, 2008.

[6] A. Arnold, J.L. López, P.A. Markowich and J. Soler, An analysis of quantum Fokker-Planck models: a Wigner function approach, Revista Matemática Iberoamericana, 20, 771-814, 2004.

[7] A. Arnold and C. Ringhofer, An operator splitting method for the Wigner-Poisson problem, SIAM J. Numer. Anal., 33, 1622-1643, 1996.

[8] A. Arnold and C. Sparber, Quantum dynamical semigroups for diffusion models with Hartree interaction, Commun. Math. Phys., 251, 179-207, 2004.

[9] D. Bohm, Quantum Theory, Courier Dover Publications, 1989.

[10] J.A. Cañizo, J.L.L. López and J. Nieto, Global $l^{1}$ theory and regularity for the $3 d$ nonlinear Wigner-Poisson-Fokker-Planck system, J. Differ. Equs., 198, 356-373, 2004.

[11] Y. Cheng and C.W. Shu, A discontinuous Galerkin finite element method for time dependent partial differential equations with higher order derivatives, Math. Comput., 77, 699-730, 2008.

[12] L. Diósi, On high-temperature Markovian equations for quantum Brownian motion, Europhysics Letters, 22, 1-3, 1993.

[13] A. Donarini, T. Novotný and A.P. Jauho, Simple models suffice for the single-dot quantum shuttle, New Journal of Physics, 7, 237-262, 2005.

[14] M. Elk and P. Labropoulos, Connection between approximate Fokker-Planck equations and the Wigner function applied to the micromaser, Quantum and Semiclassical Optics, 8, 23-37, 1996.

[15] I.M. Gamba and J. Proft, Stable discontinuous Galerkin schemes for linear Vlasov-Boltzmann transport equations, submitted.

[16] J.L. García-Palacios, Solving quantum master equations in phase space by continued-fraction methods, arXiv, 2004.

[17] J.L. García-Palacios and D. Zueco, Caldeira-Leggett quantum master equation in Wigner phase space: continued-fraction solution and application to Brownian motion in periodic potentials, Journal of Physics A: Mathematical and General, 37, 10735-10770, 2004.

[18] S. Gottlieb and C.W. Shu, Total variation diminishing Runge-Kutta schemes, Math. Comput., $67,73-85,1998$.

[19] T. Goudon, Analysis of a semidiscrete version of the wigner equation, SIAM J. Numer. Anal., 40, 2007-2025, 2002.

[20] R. Kosik, Numerical Challenges on the road to NanoTCAD, PhD thesis, Technischen Universität Wien, 2004.

[21] G. Lindblad, On the generators of quantum mechanical semigroups, Commun. Math. Phys., 48, 119-130, 1976.

[22] C. Manzini and L. Barletti, An analysis of the Wigner-Poisson problem with inflow boundary conditions, Nonlinear Analysis, 60, 77-100, 2005.

[23] B. Rivière, M.F. Wheeler and V. Girault, A priori error estimates for finite element methods based on discontinuous approximation spaces for elliptic problems, SIAM J. Numer. Anal., 39, 902-931, 2001.

[24] C.W. Shu and S. Osher, Efficient implementation of essentially nonoscillatory shock-capturing schemes, J. Chem. Phys., 77, 439-471, 1988.

[25] C. Sparber, J.A. Carillo, J. Dolbeault and P.A. Markowich, On the long-time behavior of the Quantum Fokker-Planck equation, Monatshefte für Mathematik, 141, 237-257, 2004.

[26] N.D. Suh and M.R. Feix, Numerical simulation of the Quantum Liouville-Poisson system, J. Comput. Phys., 94, 403-418, 1991. 
[27] E.P. Wigner, On the quantum correction for thermodynamic equilibrium, Physical Reviews, 40, 749-759, 1932.

[28] L. Yuan and C.W. Shu, Discontinuous Galerkin method based on non-polynomial approximation spaces, J. Comput. Phys., 218, 295-323, 2006. 